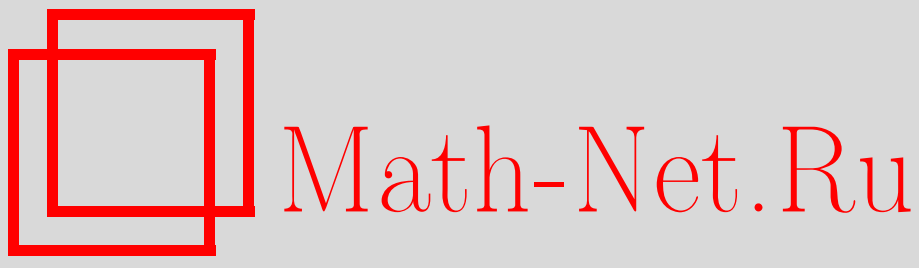

А. Л. Лукашов, Круговые параметры многочленов, ортогональных на нескольких дугах единичной окружности, Матем. сб., 2004, том 195, номер 11, 95-118

DOI: https://doi.org/10.4213/sm860

Использование Общероссийского математического портала Math-Net.Ru подразумевает, что вы прочитали и согласны с пользовательским соглашением

http://www . mathnet.ru/rus/agreement

Параметры загрузки:

IP: 54.162 .85 .209

26 апреля 2023 г., $18: 15: 02$ 
УДК 517.5

\author{
А. Л. Лукашов
}

\title{
Круговые параметры многочленов, ортогональных на нескольких дугах единичной окружности
}

\begin{abstract}
Исследуется асимптотическое поведение круговых параметров $\left(a_{n}\right)$ многочленов, ортогональных на единичной окружности относительно мер Геронимуса. Доказано, что лишь при рациональности гармонических мер дуг, составляющих носитель меры ортогональности, соответствующие круговые параметры образуют, начиная с некоторого номера, псевдопериодическую последовательность (т.е. после подходящего поворота окружности и соответствующего изменения мер ортогональности они образуют периодическую последовательность). Кроме этого установлено, что если гармонические меры этих дуг линейно независимы над полем рациональных чисел, то множества предельных точек последовательностей модулей круговых параметров $\left|a_{n}\right|$ и их отношений $\left(a_{n+k} / a_{n}\right)_{n=1}^{\infty}$ являются соответственно отрезком и континуумами комплексной плоскости.

Библиография: 43 названия.
\end{abstract}

\section{§1. Введение}

Теория ортогональных многочленов на единичной окружности $\Gamma=\{z:|z|=1\}$, т.е. многочленов $P_{n}(z)=z^{n}+\cdots$, удовлетворяюших соотношениям

$$
\int_{0}^{2 \pi} P_{n}\left(e^{\mathrm{i} \varphi}\right) \overline{P_{m}\left(e^{\mathrm{i} \varphi}\right)} d \sigma(\varphi)=\delta_{n, m} h_{n}, \quad h_{n}>0, \quad n, m \in \mathbb{N}
$$

созданная в работах Г. Сегё и Я. Л. Геронимуса (см. [1], [2]), привлекает большое внимание исследователей как благодаря своим связям с другими областями математики, так и из-за многочисленных приложений (см., например, [3] и упомянутую там литературу). Первоначально наиболее исследованным был случай мер, удовлетворяющих условию Сегё $\log \sigma^{\prime} \in L_{1}(\Gamma)$, впоследствии это условие ослаблялось в двух основных направлениях: изменение метрических свойств мер ортогональности с сохранением в качестве носителя всей окружности (укажем здесь фундаментальные работы Е. А. Рахманова и В. М. Бадкова [4], [5]) и сужение носителя меры. Тесно связанной с теорией ортогональных многочленов на окружности является и теория ортогональных многочленов на отрезке, многие достижения в которой (как и в теории ортогональных многочленов относительно неэрмитовых

Работа выполнена при частичной поддержке Российского фонда фундаментальных исследований (грант № 04-01-00060), Программы “Университеты России" (грант № УР.04.01.040), Программы поддержки ведущих научных школ РФ (грант № НШ-1295.2003.1) и Австрийского научного фонда FWF (грант № P16390-T04). 
скалярных произведений) получались в рамках теории диагональных аппроксимаций Паде. Доказательства некоторых из них содержат идеи, близкие к использованным в настояшей работе (см. обзор [6]).

Первыми работами, в которых рассматривались веса на более узких множествах, чем окружность, были работы Я.Л. Геронимуса [7] и Н.И. Ахиезера [8]. В [7; теорема X] многочлены, ортогональные на нескольких дугах (с возможным добавлением к абсолютно непрерывной мере ортогональности $\mu, d \mu=w\left(e^{\mathrm{i} \varphi}\right) d \varphi$, конечного числа масс специального вида в точках вне системы дуг), появились при изучении мер, соответствующих периодической последовательности круговых параметров (или параметров Я.Л. Геронимуса [9] $a_{n} \stackrel{\text { def }}{=}-\overline{P_{n+1}(0)}$ ), а в [8] были опубликованы (без доказательства) обобшения ряда результатов Сегё на случай одной дуги единичной окружности. Позже в [10] эти утверждения были перенесены (также без доказательства) на случай нескольких дуг. Подробные доказательства указанных результатов Н. И. Ахиезера [8] были опубликованы совсем недавно [11].

Серьезным продвижением в данном направлении послужила глубокая работа Г. Видома [12], в которой был получен ряд результатов об эрмитово ортогональных многочленах не только на нескольких отрезках или дугах, но и на сушественно более общих множествах комплексной плоскости, а также решены многие тесно связанные с ними задачи. Тем не менее некоторые задачи, вполне аналогичные ранее решенньм классическим задачам для многочленов, ортогональных на всей окружности или на отрезке, оставались нерешенными. Так, в 1984 году А.И. Аптекарев [13], развивая подход Г. Видома, нашел новые, более “явные”, асимптотические формулы для многочленов, ортогональных на системе жордановых кривых и дуг (с использованием тэта-функций Римана). С помощью найденных формул там же были получены асимптотики для многочленов, ортогональных на системе отрезков $E$ действительной оси, позволившие в связи с решением некоторых задач из теории дискретных операторов Штурма-Лиувилля найти геометрическую характеристику носителей мер, допускаюших периодические (точнее, даже предельно-периодические) последовательности коэффициентов трехчленных рекуррентных соотношений.

Указанная характеристика появлялась ранее в работе [12] для других задач (например, для конечности множества предельных точек последовательности $\left\|P_{n}\right\|_{L^{2}(E)}(C(E))^{2 n}$, где $C(E)-($ логарифмическая) емкость $E)$. Для той же последовательности у Г. Видома был описан другой экстремальньй случай - когда ее множество предельных точек заполняет цельй промежуток. Аналогичное поведение предельных точек последовательностей коэффициентов трехчленных рекуррентных соотношений было описано сушественно позже: в [14] для двух отрезков ( с использованием представлений этих коэффициентов в эллиптических функциях) и в [15] для обшего случая нескольких отрезков действительной оси (с использованием представлений через автоморфные функции). Отметим также работу [16], посвященную исследованию диагональных аппроксимаций Паде (а следовательно, и ортогональных многочленов) для класса мер, включающего и рассмотренные в [14], [15]. Заметим здесь также, что квазипериодичность этих последовательностей легко следует из результатов работ [17], [18]. (Напомним, что последовательность $f(n)$ квазипериодична, если $f(x)$ может быть представлена в виде 
$f(x)=F\left(\omega_{1} x, \ldots, \omega_{k} x\right)$, где $F-$ непрерывная 1-периодическая по каждой переменной функция.)

Для нескольких дуг единичной окружности аналогичная теория стала интенсивно развиваться после появления работы $\Phi$. Пехерсторфера и Р. Штейнбауэра [19], [20], при этом, в отличие от случая всей окружности, где переход с отрезка на окружность не только не представляет существенных трудностей, но и наоборот проясняет ситуацию (например для весов якобиевого типа В. М. Бадков [5] получал результаты именно на окружности, затем перенося их на отрезок), для нескольких дуг соответствующий переход, как отмечено в [10], представляет дополнительные трудности. Кроме того, не всякая система дуг единичной окружности может быть получена преобразованием системы отрезков действительной оси с помощью функции Жуковского, как это обьчно делается для всей окружности, поэтому имеющиеся результаты для нескольких отрезков не могут быть перенесены этой заменой переменного на случай нескольких дуг.

Цель настоящей работы - получить для случая нескольких дуг аналоги упомянутых утверждений А. И. Аптекарева, $Ф$. Пехерсторфера и автора, т.е. изучить поведение последовательностей модулей $\left|a_{n}\right|$ и частных $a_{n+1} / a_{n}$ круговых параметров ортогональных многочленов относительно мер Я. Л. Геронимуса (точные определения см. ниже) с помощью функций Шоттки-Бернсайда. Отметим здесь также, что поведение последовательностей круговых параметров во многом определяет поведение самих ортогональных многочленов, с чем связано огромное количество работ, посвященных изучению классов ортогональных многочленов, определяемых с помошью различных ограничений на эти параметры (см., например, [9], [21] и указанную там литературу).

Основным инструментом исследований будут функции Шоттки-Бернсайда. Впервые подобные функции были использованы в теории приближений в работе Н. И. Ахиезера [22], сходная техника использовалась в [23], [24; гл. 5], [25], причем в двух последних работах была продемонстрирована эффективность ее применения и для практических вычислений.

Автор выражает благодарность $\Phi$. Пехерсторферу за организацию визитов в университет И. Кеплера, г. Линц, и полезные дискуссии, а также А.И. Аптекареву и другим участникам семинара А. А. Гончара за конструктивное обсуждение приводимых результатов. Особенно благодарен автор А. Б. Богатырёву и С. П. Суетину, высказавшим ряд ценных советов и замечаний, способствовавших улучшению первоначального варианта работы и исправлению допущенных неточностей.

\section{§2. Обозначения и формулировка результатов}

Пусть $E=\bigcup_{j=1}^{l}\left[\varphi_{2 j-1}, \varphi_{2 j}\right], \varphi_{1}<\cdots<\varphi_{2 l}, \varphi_{2 l}-\varphi_{1}<2 \pi,-$ заданная фиксированная система отрезков, $\Gamma_{E}=\left\{z \in \mathbb{C}: z=e^{\mathrm{i} \varphi}, \varphi \in E\right\}$ - соответствующая система дуг единичной окружности.

Основным объектом исследования настоящей работы являются многочлены, ортогональные относительно мер Я. Л. Геронимуса [7]. Класс таких мер включает в себя все меры, для которых круговые параметры образуют, начиная с некоторого номера, непостоянную периодическую последовательность. 
Итак, пусть

$$
R(z)=c_{R} \prod_{j=1}^{2 l}\left(z-e^{\mathrm{i} \varphi_{j}}\right)
$$

- самовзаимный (т.е. такой, что $R=R^{*}$, где через $R^{*}(z)$ обозначается взаимный к $R$ многочлен, $\left.R^{*}(z)=z^{2 l} \overline{R(1 / \bar{z})}\right)$ задающий систему отрезков $E$ многочлен, $A(z)=c_{A} \prod_{j=1}^{m}\left(z-z_{j}\right)^{m_{j}}$ - фиксированный алгебраический многочлен степени $2 a \in \mathbb{Z}_{+}$, не обращающийся в нуль на $\Gamma_{E}$ и удовлетворяющий равенству $A=-A^{*}$. Далее, пусть

$$
R(z)=W(z) V(z)
$$

- некоторое разложение многочлена $R(z)$ на самовзаимные многочлены $W$ и $V$ степеней $2 w \in \mathbb{Z}_{+}$и $2 v \in \mathbb{Z}_{+}$соответственно ( $w$ и $v$, вообще говоря, - полуцелые числа),

$$
\lambda=\left(\lambda_{1}, \ldots, \lambda_{m}\right)
$$

- фиксированньй вектор такой, что $\lambda_{j} \in\{+1,-1\}, j=1, \ldots, m$, причем $\lambda_{j}$ может быть равно -1 лишш для простых нулей $z_{j}=e^{\mathrm{i} \psi_{j}}$ многочлена $A$, расположенных на $\Gamma \backslash \Gamma_{E}$. Меры Геронимуса - это меры вида

$$
d \sigma(\varphi)=d \sigma(\varphi ; A, W, \lambda)=f(\varphi ; A, W) d \varphi+\pi \sum_{j=1}^{m} \mu_{j}\left(1-\lambda_{j}\right) \delta\left(e^{\mathrm{i} \varphi}-z_{j}\right)
$$

где

$$
f(\varphi ; A, W)= \begin{cases}\left|\frac{W\left(e^{\mathrm{i} \varphi}\right)}{A\left(e^{\mathrm{i} \varphi}\right) \sqrt{\left|R\left(e^{\mathrm{i} \varphi}\right)\right|}}\right|, & \varphi \in E, \\ 0, & \varphi \notin E,\end{cases}
$$

причем $A$ и $W$ таковы, что

$$
\begin{gathered}
\operatorname{sign} \frac{\mathrm{i} W\left(e^{\mathrm{i} \varphi}\right)}{A\left(e^{\mathrm{i} \varphi}\right)} e^{\mathrm{i}(a-w) \varphi}=(-1)^{j}, \quad \varphi \in\left(\varphi_{2 j-1}, \varphi_{2 j}\right), \quad j=1, \ldots, l ; \\
\mu_{j}=\left|\frac{W\left(z_{j}\right)}{A^{\prime}\left(z_{j}\right) \sqrt{\mid R\left(z_{j}\right)} \mid}\right|
\end{gathered}
$$

и $\delta\left(z-z_{j}\right)-\delta$-функция Дирака с носителем в точке $z=z_{j}$. Кроме того, степени многочленов таковы, что $a+l / 2-w-$ натуральное число.

Таким образом, основным объектом изучения в настоящей работе будут многочлены $P_{n}$ с единичным старшим коэффициентом, ортогональные относительно мер $d \sigma(\varphi)$, т.е. удовлетворяющие равенствам

$$
\int_{0}^{2 \pi} P_{n}\left(e^{\mathrm{i} \varphi}\right) \overline{P_{m}\left(e^{\mathrm{i} \varphi}\right)} d \sigma(\varphi)=\delta_{n, m} h_{n}, \quad h_{n}>0, \quad n, m \in \mathbb{Z}_{+} .
$$

В работе будут получены и использованы явные представления этих многочленов в терминах функций Шоттки-Бернсайда.

Несколько слов об этом термине. В работах [22], [26] рассматриваемые функции называются функциями Шоттки, в известном справочнике Бейтмена-Эрдейи 
- функциями Бернсайда. Представляется исторически более целесообразным предлагаемое вьше название, поскольку В. Бернсайду [27] принадлежит важное утверждение, которое далее будет именоваться теоремой Бернсайда о представлении, позволяюшее находить формулы для автоморфных функций с заданньми нулями и полюсами, доказанное с помошью замеченной им связи теории автоморфных функций и теории абелевых интегралов. Поскольку помимо указанных классических сочинений имеются и более современные изложения теории функций Шоттки-Бернсайда [24], [25], ограничимся кратким перечислением основных обозначений и определений.

Итак, рассмотрим фундаментальную область $\mathbb{T}$ группы Шоттки, состоящую из внешности кругов $K_{1}, \ldots, K_{l-1}, K_{1}^{\prime}, \ldots, K_{l-1}^{\prime}$, расположенных вне друг друга так, что их центры лежат на мнимой оси, а также $\partial K_{1}, \ldots, \partial K_{l-1}$. Круги $K_{1}, \ldots, K_{l-1}$ расположены в верхней полуплоскости, а круги $K_{1}^{\prime}, \ldots, K_{l-1}^{\prime}$ симметричны $K_{1}, \ldots, K_{l-1}$ соответственно относительно действительной оси. Соответствуюшая группа Шоттки $\mathfrak{G}=\mathfrak{G}\left(K_{1}, \ldots, K_{l-1}\right)$ является свободной группой с операцией суперпозиции и образующими $T_{1}, \ldots, T_{l-1}$, где $T_{i}-$ дробно-линейное отображение, переводяшее внешность круга $K_{i}^{\prime}$ на внутренность круга $K_{i}$ и являющееся суперпозицией инверсии относительно окружности $\partial K_{i}^{\prime}$ и симметрии относительно действительной оси. Занумеруем отображения, входящие в группу $\mathfrak{G}$, каким-то образом, причем будем считать, что $T_{0}(z) \equiv z$,

$$
T_{i}(z)=\frac{R_{i}^{2}}{z-\bar{c}_{i}}+c_{i}, \quad i=1,2, \ldots
$$

В дальнейшем удобно использовать также обозначения Бернсайда (употреблявшиеся и в [26; гл. 12]) $c_{i^{-1}}=T_{i}^{-1}(\infty)=\bar{c}_{i}, c_{i^{-1} j}=T_{i}^{-1}\left(T_{j}(\infty)\right)$. Далее, нормированная форма отображений $T_{i}$ будет иметь вид $T_{i}(z)=\left(\alpha_{i} z+\beta_{i}\right) /\left(\gamma_{i} z+\delta_{i}\right)$, $\alpha_{i} \delta_{i}-\beta_{i} \gamma_{i}=1, \operatorname{Re} \alpha_{i}>0$. В работе [28] была доказана равномерная сходимость внутри $\mathbb{T}$ в рассматриваемой ситуации квадратных тэта-рядов Пуанкаре

$$
\theta(z, a)=\sum_{i=0}^{\infty} \frac{\left(\gamma_{i} z+\delta_{i}\right)^{-2}}{\left(\alpha_{i} z+\beta_{i}\right) /\left(\gamma_{i} z+\delta_{i}\right)-a} .
$$

С помощью тэта-рядов там же были определены постоянные

$$
a_{p q}=\int_{A_{q}^{\prime} A_{q}} \theta\left(z, c_{p}\right) d z
$$

где интегрирование производится по путям $A_{q}^{\prime} A_{q}$, не пересекаюшим ни самих себя, ни друг друга, находящимся вне кругов $K_{1}, \ldots, K_{l-1}, K_{1}^{\prime}, \ldots, K_{l-1}^{\prime}$ и соединяющим точки $A_{q}=c_{q}+\mathrm{i} R_{q}$ и $A_{q}^{\prime}=\bar{c}_{q}-\mathrm{i} R_{q}, p, q=1, \ldots, l-1$. Определим теперь функции Шоттки-Бернсайда $\Omega(z, y)$ и $\Phi_{k}(z)$ (сохраняя обозначения В. Бернсайда)

$$
\begin{gathered}
\Omega(z, y)=(z-y) \prod_{k}^{\prime} \frac{\left(T_{k}(z)-y\right)\left(T_{k}(y)-z\right)}{\left(T_{k}(z)-z\right)\left(T_{k}(y)-y\right)}, \\
\exp \Phi_{k}(z)=\frac{z-c_{k}}{z-c_{k-1}} \prod_{\substack{j=1 \\
j \neq k}}^{\infty} \frac{z-c_{j-1} k}{z-c_{j-1}}, \quad k=1, \ldots, l-1,
\end{gathered}
$$


где $z, y \in \bigcup_{T \in \mathfrak{G}} T(\mathbb{T})$ и штрих у знака произведения в $(7)$ означает, что из каждой пары взаимно обратных отображений берется лишь одно и $k>0$. Сходимость произведений в (7) и (8) следует из сходимости ряда (5) и доказана, например, в [28]. При этом соотношением $(8)$ функции $\Phi_{k}(z)$ определены однозначно по модулю $2 \pi \mathrm{i}$. Заметим, что в [28; с. 64] функции $\Phi_{k}(z)$ определяются (для любого $k \in \mathbb{N}$ ) как $\int_{z_{0}}^{z} \theta\left(z, c_{k}\right) d z$, где в качестве $z_{0} \in \mathbb{T}$ берется произвольная фиксированная точка (для определенности возьмем $z_{0}=\infty$ ), а интегрирование производится по пути, лежашему в $\mathbb{T}$ с разрезами вдоль путей $A_{q}^{\prime} A_{q}, q=1, \ldots, l-1$. При этом в [28; с. 63] было доказано, что интеграл в (6) не зависит от того, какая именно пара соответствующих при отображении $T_{q}$ точек $A_{q}, A_{q}^{\prime}$ взята на окружностях $\partial K_{q}, \partial K_{q}^{\prime}$.

Нетрудно показать (см. также лемму 2 ниже), что для любой системы дуг $\Gamma_{E}$ найдется область $\mathbb{T}_{+}=\mathbb{T} \cap\{\operatorname{Im} z>0\}$, которую можно конформно отобразить на $\widehat{\mathbb{C}} \backslash \Gamma_{E}$. Обозначим через $\phi(u)$ это отображение, представление которого через функции Шоттки-Бернсайда будет дано ниже в лемме 3 . Через $v_{j}, \xi, u_{j}$ будем обозначать соответственно прообразы $z_{j}, \infty$ и тех из точек $\exp \left(\mathrm{i} \varphi_{j}\right)$, которые являются нулями $W$, при отображении $\phi$. Напомним также, что числа $x_{1}, \ldots, x_{n}$ называются линейно независимьми над полем рациональных чисел, если равенство $k_{1} x_{1}+\cdots+k_{n} x_{n}=0$, где $k_{1}, \ldots, k_{n}$ рациональные, возможно лишш при $k_{1}=\cdots=k_{n}=0$, а последовательность $\left\{a_{n}\right\}$ псевдопериодическая, если для некоторого $k \in \mathbb{N}$ и $\alpha \in \Gamma$ и для всех $n>n_{0} \quad a_{n+k}=\alpha^{2} a_{n}, \alpha=e^{-\mathrm{i} k \theta / 2}$. Нам также понадобится двулистная риманова поверхность $\mathscr{S}$ функции $\sqrt{R(z)}$, линиями перехода которой являются дуги $\Gamma_{E}$. Через $\omega_{j}(z), j=1, \ldots, l$, будем обозначать гармонические меры дуг $\Gamma_{j}=\left\{\exp (\mathrm{i} \varphi): \varphi \in\left[\varphi_{2 j-1}, \varphi_{2 j}\right]\right\}$ относительно области $\mathbb{C} \backslash \Gamma_{E}$ (в точке $\left.z \in \mathbb{C} \backslash \Gamma_{E}\right) ;$ иными словами, $\omega_{j}(z)$-гармоническая в $\mathbb{C} \backslash \Gamma_{E}$ функция, граничные значения которой равны единице на $\Gamma_{j}$ и нулю на $\Gamma_{E} \backslash \Gamma_{j}$. Напомним также, что задание последовательности круговых параметров $\left\{a_{n}\right\},\left|a_{n}\right|<1$, однозначно определяет последовательность ортогональных на единичной окружности многочленов $P_{n}$ с единичным старшим коэффициентом и, следовательно, меру ортогональности [29].

Основным результатом работы является следующая

ТЕОРема 1. а) Последовательность круговых параметров $\left\{a_{n}\right\},\left|a_{n}\right|<1$, является псевдопериодической тогда и только тогда, когда отвечающие ей многочлень ортогональны относительно некоторой меры вида (2), а гармонические меры $\omega_{j}(\infty), j=1, \ldots, l$, дуг $\Gamma_{j}$, составляющих мнохсество $\Gamma_{E},-$ рациональные числа.

б) Если система дуг $\Gamma_{E}$ такова, что гармонические меры $\omega_{j}(\infty), j=$ $1, \ldots, l$, для множества $\Gamma_{E}$ линейно независимы над полем рациональных чисел, то для любой меры вида (2) с R(z), заданным системой E, последовательность $\left\{\left|a_{n}\right|\right\}$ и любая последовательность $\left\{a_{n+k} / a_{n}\right\}, k \in \mathbb{N}$, имеют своими множсествами предельных точек отрезок и континуум комплексной плоскости соответственно.

ЗАмЕчАниЕ 1. В [30; теорема 4.2] содержится утверждение, совпадающее с п. а) теоремы 1 , но с заменой псевдопериодичности на периодичность, хотя по сути доказано было именно сформулированное здесь утверждение. $\Phi$. Пехерсторфер любез- 
но сообщил автору, что теорема 4.2 из [30] будет верной, если в (ii), (iii) положить $\beta=1$, а в (iv) добавить "and $R^{\circ}(0)=1$."

\section{§3. Вспомогательные результаты}

Прежде всего приведем необходимые для дальнейшего свойства функций Шоттки-Бернсайда. Во-первых, при действиях подстановок соответствующей группы Шоттки $\mathfrak{G}$ функции $\Omega$ и $\Phi$ ведут себя следуюшим образом:

$$
\begin{gathered}
\frac{\Omega\left(T_{p}(z), y\right)}{\Omega(z, y)} \equiv \epsilon_{p}\left(\gamma_{p} z+\delta_{p}\right)^{-1} \exp \left\{\Phi_{p}(y)-\Phi_{p}(z)-\frac{a_{p p}}{2}\right\} \\
\frac{\exp \Phi_{i}\left(T_{p}(z)\right)}{\exp \Phi_{i}(z)} \equiv \exp a_{i p},
\end{gathered}
$$

$i, p=1, \ldots, l-1$, где $\epsilon_{p}= \pm 1$ зависят лишш от области $\mathbb{T}$ и выбранной нормировки отображений $T_{i}$. (Методом работы [26; с. 360-363] можно показать, что здесь $\epsilon_{p}=-1, p=1, \ldots, l-1$, но этот факт не будет использоваться в дальнейшем.) Соотношения $(9),(10)$ приводятся (с некоторыми неточностями, на которые внимание автора обратил А.Б. Богатырёв) в [27], [28] (см. также [26]). Кроме этих свойств нам потребуется еше ряд соотношений, доказанных в [27], [28]:

1) $a_{p q}=a_{q p}$ для любых $1 \leqslant p, q \leqslant l-1$,

2) $a_{p q} \in \mathbb{R}$ в рассматриваемом (симметричном) случае, $1 \leqslant p, q \leqslant l-1$,

3) $\Omega(z, y) \equiv-\Omega(y, z)$,

а также теорема Бернсайда о представлении, по существу доказанная в [27].

ТЕОРема 2. Если автоморфная относительно группь $\mathfrak{G}$ функиия $f$ имеет нули $y_{1}, \ldots, y_{n}$ и полюсы $x_{1}, \ldots, x_{n}$ в фундаментальной области $\mathbb{T}$, то

$$
f(z)=\mathrm{const} \cdot \frac{\Omega(z, \zeta)}{\Omega\left(z, x_{n}\right)} \prod_{j=1}^{n-1} \frac{\Omega\left(z, y_{j}\right)}{\Omega\left(z, x_{j}\right)},
$$

где $\zeta-$ образ точки $y_{n}$ при некотором отображсении группъ $\mathfrak{G}$.

Нам понадобятся некоторые дополнительные свойства функций $\Omega$ и $\Phi$.

Лемма 1. Для любых точек $z, y \in \mathbb{T} u k, p=1, \ldots, l-1$, справедливы соотношения

$$
\begin{gathered}
\exp \Phi_{k}(-z)=\exp \left(-\Phi_{k}(z)\right), \\
\exp \Phi_{k}(-\bar{z})=\exp \overline{\Phi_{k}(z)}, \\
\Omega(-\bar{z}, y)=-\overline{\Omega(z,-\bar{y})} \\
\Omega(-z, y)=-\Omega(z,-y), \\
\Delta_{z \in \partial K_{k}} \arg \exp \Phi_{p}(z)=2 \pi \delta_{p k}, \\
\Delta_{z \in \partial K_{k}} \arg \Omega(z, y)=0 \quad \text { для } \quad y \in \mathbb{T} .
\end{gathered}
$$

Здесь $\Delta \arg$ обозначает изменение аргумента при обходе соответствующего контура против часовой стрелки, а $\delta_{p k}-$ символ Кронекера. 
ДокАЗАТЕЛЬСТво. В силу (4), (8)

$$
\begin{aligned}
\exp \Phi_{k}(-z) & =\frac{z-\bar{c}_{k}}{z-c_{k}} \prod_{\substack{j=1 \\
j \neq k}}^{\infty} \frac{z+T_{j}^{-1}\left(T_{k}(\infty)\right)}{z+T_{j}^{-1}(\infty)} \\
& =\frac{z-c_{k}-1}{z-c_{k}} \prod_{\substack{j=1 \\
j \neq k}}^{\infty} \frac{z-T_{j}\left(T_{k}^{-1}(\infty)\right)}{z-T_{j}(\infty)}=\exp \Phi_{p}(z)
\end{aligned}
$$

где $p$ - такой индекс, что $T_{p}=T_{k}^{-1}$. Но

$$
\Phi_{p}(z)=\int_{\infty}^{z} \theta\left(z, c_{p}\right) d z=-\int_{\infty}^{z} \theta\left(z, c_{j}\right) d z=-\Phi_{j}(z)
$$

Таким образом, (11) доказано. Формула (12) доказывается аналогично. Проверим (13):

$$
\Omega(-\bar{z}, y)=(-\bar{z}-y) \prod_{j}^{\prime} \frac{\left(T_{j}(-\bar{z})-y\right)\left(T_{j}(y)+\bar{z}\right)}{\left(T_{j}(-\bar{z})+\bar{z}\right)\left(T_{j}(y)-y\right)}
$$

откуда, последовательно применяя очевидное тождество $T_{j}(-\bar{z})=-\overline{T_{j}(z)}, j=$ $1,2, \ldots$, получим требуемое. Тождество (14) доказьвается аналогично с учетом того, что для $j=1, \ldots, l-1 T_{j}(-z)=-T_{j}^{-1}(z)$. Равенства $(15),(16)$ проверяются непосредственным подсчетом изменения аргумента с учетом того, что аргументы всех сомножителей в бесконечных произведениях в $(8),(7)$ не изменяются при обходе вдоль граничных окружностей $\partial K_{k}$.

Следуюшая лемма доказана в [19], она является основой для получения представлений рассматриваемых ортогональных многочленов, поскольку позволяет свести нахождение ортогональных многочленов к решению алгебраического уравнения типа уравнения Пелля в теории чисел. При этом условия (18)-(20) фактически определяют компоненты дивизора мероморфной функции на римановой поверхности $\mathscr{S}$, расположенные над точками $z_{j}, j=1, \ldots, m, \infty$ и 0, а над нулями многочлена $g_{(n)}($ см. $(18))$ при этом будут располагаться неизвестные компоненты дивизора, асимптотическое поведение которых исследуется в работе сведением к проблеме обрашения Якоби. При этом здесь и далее выбирается ветвь квадратного корня так, чтобы

$$
\arg \sqrt{R\left(e^{\mathrm{i} \varphi}\right)}=\arg (-1)^{j} e^{\mathrm{i} l \varphi / 2}, \quad \varphi \in\left(\varphi_{2 j}, \varphi_{2 j+1}\right), \quad j=1, \ldots, l-1 .
$$

Лемма 2. Пусть $d \sigma-$ мера вида (2), $n \geqslant a+l / 2+v, P_{n}$ - многочлен степени п. Тогда следующие утвержсдения эквивалентны:

1. Многочлен $P_{n}$ ортогонален относительно меры $d \sigma$;

2. Найдутся многочлены $Q_{n+l-2 v}$ u $g_{(n)}$ степеней $n+l-2 v$ ul-1 соответственно такие, что

$$
W(z) P_{n}^{2}(z)-V(z) Q_{n+l-2 v}^{2}(z)=z^{n-(a+l / 2-w)} A(z) g_{(n)}(z),
$$


причем $g_{(n)}(z)$ может обращаться в нуль в точке $z=0$ тогда и только тогда, когда $P_{n}(0)=0$. $B$ этом случае кратности нуля $P_{n}$ $u g_{(n)}$ в $z=0$ совпадают $u$

$$
\begin{gathered}
\left(V Q_{n+l-2 v}\right)^{(i)}\left(z_{j}\right)=\lambda_{j}\left(\sqrt{R} P_{n}\right)^{(i)}\left(z_{j}\right), \\
j=1, \ldots, m, \quad i=0, \ldots, m_{j}-1, \\
\left.\frac{V Q_{n+l-2 v}}{\sqrt{R} P_{n}}\right|_{z=0}=\left.\frac{V Q_{n+l-2 v}}{\sqrt{R} P_{n}}\right|_{z=\infty}=1 .
\end{gathered}
$$

Следуюшая лемма описывает отображающую функцию.

Лемма 3. Отображающая функция $z=\phi(и)$ области вида $\mathbb{T}_{+}$на $\overline{\mathbb{C}} \backslash \Gamma_{E}$ имеет вид

$$
\phi(u)=C_{\phi} \frac{\Omega(u, \bar{\xi}) \Omega(u,-\bar{\xi})}{\Omega(u, \xi) \Omega(u,-\xi)},
$$

2дe

$$
C_{\phi}=e^{\mathrm{i} \varphi_{2}} \frac{\Omega(0,-\xi) \Omega(0, \xi)}{\overline{\Omega(0,-\xi) \Omega(0, \xi)}}, \quad\left|C_{\phi}\right|=1 .
$$

Функиии $g_{(n)}(\phi(u)), W(\phi(u)), A(\phi(u))$ допускают следующие представления:

$$
\begin{aligned}
g_{(n)}(\phi(u)) & =\mathrm{const} \cdot \prod_{j=1}^{l-1} \frac{\Omega\left(u, b_{j}^{(n)}\right) \Omega\left(u,-b_{j}^{(n)}\right)}{\Omega(u, \xi) \Omega(u,-\xi)}, \\
W(\phi(u)) & =\mathrm{const} \cdot \prod_{j=1}^{2 w} \frac{\Omega\left(u, u_{j}\right) \Omega\left(u,-u_{j}\right)}{\Omega(u, \xi) \Omega(u,-\xi)}, \\
A(\phi(u)) & =\mathrm{const} \cdot \prod_{j=1}^{m}\left(\frac{\Omega\left(u, v_{j}\right) \Omega\left(u,-v_{j}\right)}{\Omega(u, \xi) \Omega(u,-\xi)}\right)^{m_{j}},
\end{aligned}
$$

где $z_{j}=\phi\left( \pm b_{j}^{(n)}\right), j=1, \ldots, l-1,-$ нули многочлена $g_{(n)}$.

ДокАЗАТЕЛЬСТво. Вид функции $\phi(u)$ может быть легко получен из [23; лемма 2] путем суперпозиции построенной там функции с дробно-линейным отображением, но для дальнейшего будет удобнее представление (21). Для его доказательства заметим, что функция $z=\phi(u)$ принимает на границе области $\mathbb{T}_{+}$значения, принадлежащие единичной окружности $\Gamma$, а значит, ее можно продолжить через границу $\mathbb{T}_{+}$по принципу симметрии Римана-Шварца. Продолженная функция будет неизменной при действиях отображений группы Шоттки $\mathfrak{G}$, т.е. автоморфной. Применяя теорему Бернсайда о представлении, с учетом леммы 1 получим требуемое. Для определенности фиксируем центр одного из кругов, скажем $c_{1}=\mathrm{i}$. Чтобы установить $(22)$, подсчитаем $\phi(0)=\exp \left(\mathrm{i} \varphi_{2}\right)$ и используем (13). Доказательства формул (23)-(25) проводятся аналогично с учетом (15), (16), при этом автоморфность этих функций вытекает из установленной автоморфности $\phi(u)$.

Следующая лемма содержит представление ортогональных многочленов через автоморфные функции, необходимое для дальнейшего. 
ЛЕмма 4. В предположсениях леммы 2 ортогональные многочлены $P_{n}$ допускают параметрическое представление

$$
\begin{gathered}
P_{n}(z)=\mathrm{const} \cdot\left(\Omega_{n}(u)+\Omega_{n}(-u)\right), \\
z=\phi(u),
\end{gathered}
$$

əдe

$$
\begin{aligned}
& \Omega_{n}(u)=\left(\frac{\Omega(u, \bar{\xi})}{\Omega(u, \xi)}\right)^{n}\left(\prod_{j=1}^{l-1} \frac{\Omega\left(u, b_{j}^{(n)}\right)}{\Omega(u,-\xi)}\right) \frac{(\Omega(u, \bar{\xi}) \Omega(u,-\xi))^{w-a+l / 2}}{\Omega^{l}(u, \bar{\xi})} \\
& \times \frac{\prod_{j=1}^{m} \Omega^{m_{j}\left(1+\lambda_{j}\right) / 2}\left(u,-v_{j}\right) \Omega^{m_{j}\left(1-\lambda_{j}\right) / 2}\left(u, v_{j}\right)}{\prod_{j=1}^{2 w} \Omega\left(u, u_{j}\right)} \exp \sum_{j=1}^{l-1} k_{j}^{(n)} \Phi_{j}(u),
\end{aligned}
$$

точки $b_{j}^{(n)} \in \mathbb{T}, j=1, \ldots, l-1$, и челье числа $k_{j}^{(n)}, j=1, \ldots, l-1$, удовлетворяют системе уравнений

$$
\begin{aligned}
& \exp \left(\left(n-a-\frac{l}{2}+w\right)\left(\Phi_{j}(\xi)-\Phi_{j}(\bar{\xi})\right)+\Phi_{j}(\xi)-\sum_{k=1}^{l-1} \Phi_{j}\left(b_{k}^{(n)}\right)+\sum_{k=1}^{2 w} \Phi_{j}\left(u_{k}\right)\right. \\
& \left.\quad+\sum_{k=1}^{m} \lambda_{k} m_{k} \Phi_{j}\left(v_{k}\right)-\sum_{i=1}^{l-1} k_{i}^{(n)} a_{j i}\right)=1, \quad j=1, \ldots, l-1
\end{aligned}
$$

Обратно, если выполнены равенства $(28)$ с некоторьми $k_{j}^{(n)} \in \mathbb{Z} u b_{j}^{(n)} \in \mathbb{T}$, то многочлен $P_{n}$, заданный формулами (26), (27), ортогонален относительно мери $d \sigma(\varphi ; A, W, \lambda)$.

ДоказАтельство. Рассмотрим функцию

$$
\Psi(z)=\frac{W(z)\left(P_{n}(z)+\sqrt{V(z) / W(z)} Q_{n+l-2 v}(z)\right)^{2}}{z^{n-(a+l / 2-w)} A(z) g_{(n)}(z)},
$$

где $Q_{n+l-2 v}$ и $g_{(n)}-$ многочлены из леммы 2. Эта функция мероморфная на римановой поверхности $\mathscr{S}:\left\{\omega^{2}=R(z)\right\}$ гиперэллиптического типа, имеющей гиперэллиптическую инволюцию $I:(z, \omega) \rightarrow(z,-\omega)$. Группа $\mathfrak{G}$ униформизирует риманову поверхность $\mathscr{S}$, поэтому функция $\Psi(\phi(u))$ будет автоморфной функцией относительно групшы $\mathfrak{G}$. При этом инволюции $I$ соответствует отображение $u \rightarrow-u$, откуда и из (18) получаем

$$
\Psi(\phi(u)) \Psi(\phi(-u)) \equiv 1
$$

Заметим, что равенство (30) означает, что если $u_{0}$ - нуль функции $\Psi(\phi(u))$, то $-u_{0}-$ полюс того же порядка и наоборот. Определим теперь с помощью (18) нули и полюсы $\Psi(\phi(u))$ :

1. Точка $u=\xi(\phi(\xi)=\infty)$ является полюсом кратности $n+1-a-l / 2+w$ (здесь используем (20)). 
2. Точка $u=-\bar{\xi}(\phi(-\bar{\xi})=0)-$ полюс кратности $n-a-l / 2+w($ с учетом $(20))$.

3. Точка $u=v_{j} \quad\left(\phi\left(v_{j}\right)=z_{j}\right)$ является нулем (соответственно полюсом) кратности $m_{j}$, если $\lambda_{j}=-1$ (соответственно $\left.\lambda_{j}=1\right), j=1, \ldots, m$ (здесь используем (19)).

4. С учетом сделанного вьше замечания из п. 1 следует, что $u=-\xi$ является нулем кратности $n+1-a-l / 2+w$.

5. Аналогично, $u=\bar{\xi}-$ нуль кратности $n-a-l / 2+w$.

6. Точка $u=-v_{j}\left(\phi\left(v_{j}\right)=z_{j}\right)$ является нулем (полюсом) кратности $m_{j}$, если $\lambda_{j}=+1\left(\lambda_{j}=-1\right), j=1, \ldots, m$.

7. Точки $u=b_{1}^{(n)}, \ldots, b_{l-1}^{(n)} \subset \mathbb{T}$ являются нулями, а $u=-b_{j}^{(n)}, j=1, \ldots, l-1$, - полюсами.

$\mathrm{K}$ п. 7 необходимы некоторые пояснения. Во-первых, $\phi\left(b_{j}^{(n)}\right)$ являются нулями многочлена $g_{(n)}$, но расположение $b_{j}^{(n)}$ в $\mathbb{T}_{+}$или в $\mathbb{T}_{-}=-\mathbb{T}_{+}$не фиксируется, а определяется в зависимости от того, нуль или полюс в $b_{j}^{(n)}$ имеет функция $\Psi$. Во-вторых, равенства $(29)$ и (30) не исключают того, что не все точки $\pm b_{j}^{(n)}$ являются нулями или полюсами $\Psi(\phi(u))$, поэтому нужно дополнительно установить, что в п. 7 имеется именно $l-1$ точек. Для этого прежде всего заметим, что $P_{n}(z)$ и $g_{(n)}(z)$ не могут иметь общих нулей в точках, отличных от нулей многочлена $A(z)$ и от $z=0$, в силу (18) и равенств

$$
Q_{n+l-2 v}(z)=\frac{i P_{n}^{[1]}(z) A(z)-P_{n}(z) B(z)}{z^{a+l / 2-w}}
$$

(см. $[19 ;(2.13)])$ и

$$
P_{n}(z) P_{n}^{[1] *}(z)+P_{n}^{*}(z) P_{n}^{[1]}(z)=\mathrm{const} \cdot z^{n}
$$

(см. $[2 ;(1.17)])$, где $P_{n}^{[1]}(z)$ - многочлены второго рода, а $B=B(\cdot ; A, W, \lambda)$ - многочлен степени не выше $2 a$ [19; теорема 2.1]. Далее, наличие кратного нуля $\alpha$ многочлена $g_{(n)}$, являюшегося нулем многочлена $P_{n}$, означало бы по [19; теорема 2.1], что многочлен $P_{n-1}(z)=P_{n}(z) /(z-\alpha)$ был бы ортогонален многочленам степени $n-1$ относительно веса $d \sigma$, что невозможно. Отметим также, что какие-то из точек $b_{1}^{(n)}, \ldots, b_{l-1}^{(n)}$ могут совпасть $\mathrm{c} \pm v_{j}$ или $\pm \bar{\xi}$, приводя к сокращению некоторых сомножителей в (31).

Из пп. 1-7, теоремы 2 и формулы (9) следует представление

$$
\begin{aligned}
\Psi(\phi(u))= & \text { const } \cdot\left[\frac{\Omega(u, \bar{\xi})}{\Omega(u,-\bar{\xi})}\right]^{n-a-l / 2+w}\left[\frac{\Omega(u,-\xi)}{\Omega(u, \xi)}\right]^{n+1-a-l / 2+w} \\
& \times \prod_{j=1}^{m}\left[\frac{\Omega\left(u,-v_{j}\right)}{\Omega\left(u, v_{j}\right)}\right]^{\lambda_{j} m_{j}} \prod_{j=1}^{l-1} \frac{\Omega\left(u, b_{j}^{(n)}\right)}{\Omega\left(u,-b_{j}^{(n)}\right)} \exp \sum_{j=1}^{l-1} \widetilde{k}_{j}^{(n)} \Phi_{j}(u),
\end{aligned}
$$

где $\widetilde{k}_{j}^{(n)}, j=1, \ldots, l-1,-$ некоторые целые числа.

Из (29) следует, что

$$
\left(P_{n}(z)+\sqrt{\frac{V(z)}{W(z)}} Q_{n+l-2 v}(z)\right)^{2}=\frac{\Psi(\phi(u))(\phi(u))^{n-a-l / 2+w} A(\phi(u)) g_{(n)}(\phi(u))}{W(\phi(u))} .
$$


Подставляя сюда (31), (21)-(25), после преобразований с учетом (9) и того факта, что для $u_{j} \in \partial K_{p} T_{p}\left(u_{j}\right)=-u_{j}$, найдем

$$
\begin{aligned}
& \left(P_{n}(z)+\sqrt{\frac{V(z)}{W(z)}} Q_{n+l-2 v}(z)\right)^{2}=\mathrm{const} \cdot\left(\frac{\Omega(u, \bar{\xi})}{\Omega(u, \xi)}\right)^{2 n} \prod_{j=1}^{l-1} \frac{\Omega^{2}\left(u, b_{j}^{(n)}\right)}{\Omega^{2}(u,-\xi)} \\
& \quad \times\left(\frac{\Omega(u, \bar{\xi})}{\Omega(u,-\xi)}\right)^{-l} \prod_{j=1}^{m} \frac{\Omega^{m_{j}\left(1+\lambda_{j}\right)}\left(u,-v_{j}\right) \Omega^{m_{j}\left(1-\lambda_{j}\right)}\left(u, v_{j}\right)}{(\Omega(u, \bar{\xi}) \Omega(u,-\xi))^{m_{j}}} \\
& \quad \times \prod_{j=1}^{2 w} \frac{\Omega(u, \bar{\xi}) \Omega(u,-\xi)}{\Omega^{2}\left(u, u_{j}\right)} \exp \sum_{j=1}^{l-1} 2 k_{j}^{(n)} \Phi_{j}(u)
\end{aligned}
$$

где $2 k_{j}^{(n)}=\widetilde{k}_{j}^{(n)}+r_{j}, j=1, \ldots, l-1$, и $r_{j}$ - число нулей $W(\phi(u))$ на $\partial K_{j+1}$.

Отсюда, полагая

$$
\Omega_{n}(u) \stackrel{\text { def }}{=} P_{n}(\phi(u))+\sqrt{\frac{V(\phi(u))}{W(\phi(u))}} Q_{n+l-2 v}(\phi(u)),
$$

получим (26) и (27). Записывая условие автоморфности функции $\Omega_{n}(u)$, с помощью (9)-(11) найдем (28). При этом именно автоморфность $\Omega_{n}$ обеспечивает четность коэффициента $2 k_{j}^{(n)}$ в $(32)$ (автоморфность $\Omega_{n}$ вместе с теоремой 2 и формулой (9) дает возможность записать представление вида $(27)$ с цельми $\left.k_{j}^{(n)}\right)$.

Обратно, из равенств (28) вытекает, что функция $\Omega_{n}(u)$, построенная по формуле (27), автоморфна относительно группы $\mathfrak{G}$ и как функция переменной $z=\phi(u)$ является рациональной функцией от переменных $z$ и $\sqrt{R(z)}$. Значит,

$$
\Omega_{n}(u)=\frac{\widetilde{P}(z)+\sqrt{R(z)} \widetilde{Q}(z)}{S(z)}
$$

где $\widetilde{P}, \widetilde{Q}, S$ - многочлены. В силу (27) полюсы функции $\Omega_{n}(u)$ находятся там, где $z=\infty$ и $W(z)=0$, поэтому $S(z)=W(z)$ и $\max (\operatorname{deg} \widetilde{P}, \operatorname{deg} \widetilde{Q}+l)-2 w=n$.

Построим теперь функцию

$$
\Psi(z)=\left(\frac{\widetilde{P}(z)+\sqrt{R(z)} \widetilde{Q}(z)}{W(z)}\right)^{2} \cdot \frac{W(z)}{z^{n-(a+l / 2-w)} A(z) g_{(n)}(z)},
$$

где $g_{(n)}(z)$ - многочлен с нулями в точках $\phi\left(b_{j}^{(n)}\right), j=1, \ldots, l-1$. Учитывая $(27)$ и лемму 3 , получим, что $\Psi(z)$ обладает нулями и полюсами, перечисленными вьшше в пп. $1-7$, и удовлетворяет тождеству (30). Отсюда немедленно следует, что $\widetilde{P}(z)$ делится на $W(z)$, т.е. $\widetilde{P}(z)=W(z) P(z)$, где $P$ - многочлен степени не выше $n$. Тождество (30) тогда позволяет утверждать, что многочлены $P$ и $\widetilde{Q}$ удовлетворяют (18). Из пп. 1,4 перечисления нулей и полюсов получаем, что $\lim _{z \rightarrow \infty}(P(z)-\sqrt{R(z)} \widetilde{Q}(z))=0$, откуда $\operatorname{deg} P=\operatorname{deg} \widetilde{Q}+l$ и, переходя к взаимным многочленам,

$$
P^{*}(0)=\sqrt{R(0)} \widetilde{Q}^{*}(0) .
$$


Из пп. 2, 5 аналогично имеем

$$
\left.\frac{P(z)}{\sqrt{R(z)} \widetilde{Q}(z)}\right|_{z=0}=1
$$

что вместе с (34) дает (20). Наконец, из пп. 3, 6 получаем (19). Тогда лемма 2 дает требуемое.

ЛЕмма 5. Пусть $a_{n}$ - круговые параметры, соответствующие последовательности многочленов $\left\{P_{n}\right\}$ с единичным стариим коэффициентом, ортогональных относительно меры $d \sigma(\varphi ; A, W, \lambda)$ вида $(2), u n \geqslant a+l / 2+v$. Тогда для них справедливо представление

$$
\begin{aligned}
& -\overline{a_{n-1}}=C_{\phi}^{n}(-1)^{w-a+l / 2}\left[\frac{\overline{\Omega(\xi,-\xi)}}{\Omega(\xi,-\xi)}\right]^{n+w-a+l / 2} \prod_{j=1}^{2 w} \frac{\Omega\left(\xi, u_{j}\right)}{\Omega\left(-\bar{\xi}, u_{j}\right)} \\
& \quad \times\left[\frac{\Omega(\xi,-\xi)}{\Omega(-\bar{\xi},-\xi)}\right]^{l-1} \prod_{j=1}^{m} \frac{\Omega^{m_{j}\left(1+\lambda_{j}\right) / 2}\left(-\bar{\xi},-v_{j}\right) \Omega^{m_{j}\left(1-\lambda_{j}\right) / 2}\left(-\bar{\xi}, v_{j}\right)}{\Omega^{m_{j}\left(1+\lambda_{j}\right) / 2}\left(\xi,-v_{j}\right) \Omega^{m_{j}\left(1-\lambda_{j}\right) / 2}\left(\xi, v_{j}\right)} \\
& \quad \times \prod_{j=1}^{l-1} \frac{\Omega\left(-\bar{\xi}, b_{j}^{(n)}\right)}{\Omega\left(\xi, b_{j}^{(n)}\right)}\left[\frac{\Omega(\xi, \bar{\xi})}{\Omega(-\bar{\xi}, \bar{\xi})}\right]^{l} \exp \sum_{j=1}^{l-1} k_{j}^{(n)}\left(\Phi_{j}(-\bar{\xi})-\Phi_{j}(\xi)\right) .
\end{aligned}
$$

ДоКАЗАТЕЛьство. Запишем ортогональные относительно меры $d \sigma(\varphi ; A, W, \lambda)$ многочлены $P_{n}$ с единичньм старшим коэффициентом в виде $P_{n}(z)=c_{n} \widetilde{P}_{n}(z)$, где $\widetilde{P}_{n}(z)=\Omega_{n}(u)+\Omega_{n}(-u), z=\phi(u)$ и $\Omega_{n}$ задана в лемме 4.

Тогда

$$
c_{n}^{-1}=\lim _{z \rightarrow \infty} \frac{\widetilde{P}_{n}(z)}{z^{n}}=\lim _{u \rightarrow \xi} \frac{\Omega_{n}(u)}{(\phi(u))^{n}} .
$$

Подставляя сюда выражения (21), (27), после несложных преобразований получаем

$$
\begin{aligned}
c_{n}=C_{\phi}^{n}\left[\frac{\Omega(\xi,-\bar{\xi})}{\Omega(\xi,-\xi)}\right]^{n} \prod_{j=1}^{l-1} \frac{\Omega(\xi,-\xi)}{\Omega\left(\xi, b_{j}^{(n)}\right)} \cdot \frac{\prod_{j=1}^{2 w} \Omega\left(\xi, u_{j}\right)}{\prod_{j=1}^{m} \Omega^{m_{j}\left(1+\lambda_{j}\right) / 2}\left(\xi,-v_{j}\right) \Omega^{m_{j}\left(1-\lambda_{j}\right) / 2}\left(\xi, v_{j}\right)} \\
\quad \times \frac{\Omega^{l}(\xi, \bar{\xi})}{[\Omega(\xi, \bar{\xi}) \Omega(\xi,-\xi)]^{w-a+l / 2}} \exp \left(-\sum_{j=1}^{l-1} k_{j}^{(n)} \Phi_{j}(\xi)\right) .
\end{aligned}
$$

Из определения круговых параметров следует, что

$$
-\overline{a_{n-1}}=P_{n}(0)=c_{n} \Omega_{n}(-\bar{\xi})
$$

Отсюда и из (27), (36) с учетом равенств

$$
\Omega(\xi,-\bar{\xi})=-\Omega(-\bar{\xi}, \xi), \quad \Omega(\xi, \bar{\xi})=\Omega(-\bar{\xi},-\xi), \quad \Omega(-\bar{\xi}, \bar{\xi})=-\overline{\Omega(\xi,-\xi)}
$$

получим требуемое. 
Лемма 6. Для любой системы дуг $\Gamma_{E}$ справедливо соотношение

$$
2 \operatorname{Re} \Phi_{p}(\xi)=\sum_{j=1}^{l-1} \omega_{j+1}(\infty) a_{j p}, \quad p=1, \ldots, l-1 .
$$

ДокАЗАТЕЛЬСтво. Аналог соотношения (37) в терминах абелевых дифференциалов на соответствуюшей римановой поверхности можно найти, например, в [6; (2.26)]. Доказательство, опирающееся на ту же схему, можно было бы провести и здесь. Поскольку нам потребуются также равенства (43), полученные далее, приведем другое доказательство. Сначала проведем его для такой системы дуг $\Gamma_{E}$, что на ней существует комплексньй $T$-многочлен с единичньм старшим коэффициентом [20], т.е. такой самовзаимный многочлен $\mathscr{T}_{N}$ степени $N$, что для некоторого самовзаимного многочлена $\mathscr{U}_{N-l}$ справедливо равенство

$$
\mathscr{T}_{N}^{2}(z)-R(z) \mathscr{U}_{N-l}^{2}=L^{2} z^{N},
$$

где $L \in \mathbb{R}, L \neq 0$.

Тогда, применяя рассуждения, аналогичные использованным при доказательстве леммы 4 , найдем, что

$$
\mathscr{T}_{N}(z)=\mathrm{const} \cdot\left(\Omega_{N}^{T}(u)+\Omega_{N}^{T}(-u)\right),
$$

где

$$
z=\phi(u), \quad \Omega_{N}^{T}(u)=\left[\frac{\Omega(u, \bar{\xi})}{\Omega(u, \xi)}\right]^{N} \exp \sum_{j=1}^{l-1} m_{j}^{(N)} \Phi_{j}(u),
$$

$m_{j}^{(N)}$ обозначает число нулей многочлена $\mathscr{T}_{N}$ на $\Gamma_{j+1}, j=1, \ldots, l-1$. При этом должно выполняться условие автоморфности функции $\Omega_{N}^{T}$, т.е. с учетом леммы 1

$$
\exp \left(N\left(\Phi_{p}(\xi)-\Phi_{p}(\bar{\xi})\right)-\sum_{j=1}^{l-1} m_{j}^{(N)} a_{j p}\right)=1, \quad p=1, \ldots, l-1,
$$

откуда

$$
2 \operatorname{Re} \Phi_{p}(\xi)=\sum_{j=1}^{l-1} \frac{m_{j}^{(N)}}{N} a_{j p}, \quad p=1, \ldots, l-1 .
$$

Заметим теперь, что, делая в равенстве (38) замену

$$
z=\frac{\zeta-\mathrm{i}}{\zeta+\mathrm{i}}, \quad \zeta=\zeta(z)
$$

мы придем к равенству

$$
\mathfrak{T}_{N}^{2}(\zeta)-\mathfrak{R}(\zeta) \mathfrak{U}_{N-l}^{2}(\zeta)=L^{2}\left(\zeta^{2}+1\right)^{N}
$$

$\left(\mathfrak{T}_{N}(\zeta(z)) \stackrel{\text { def }}{=} \mathscr{T}_{N}(z)(\zeta+\mathrm{i})^{N}, \mathfrak{R}(\zeta(z)) \stackrel{\text { def }}{=} \mathscr{R}(z)(\zeta+\mathrm{i})^{2 l}, \mathfrak{U}_{N-l}(\zeta(z)) \stackrel{\text { def }}{=} \mathscr{U}_{N-l}(z) \times\right.$ $\left.(\zeta+\mathrm{i})^{N-l}\right)$, из которого следует с учетом перемежаемости нулей $\mathfrak{T}_{N}$ и $\mathfrak{U}_{N-l}$, что 
$\mathfrak{T}_{N}(\zeta) / \sqrt{\left(\zeta^{2}+1\right)^{N}}$ наименее уклоняется от нуля на $E_{\zeta}=\zeta\left(\Gamma_{E}\right) \subset \mathbb{R}$ среди всех алгебраических дробей соответствующего вида (см., например, [31]).

Тогда по теореме 3.3.6 из [32]

$$
\lim _{N \rightarrow \infty} \frac{m_{j}^{(N)}}{N}=\mu_{w}\left(E_{j+1}^{(\zeta)}\right),
$$

где $E_{j}^{(\zeta)}-$ образ дуги $\Gamma_{j}=\left\{\exp (\mathrm{i} \varphi): \varphi \in\left[\varphi_{2 j-1}, \varphi_{2 j}\right]\right\}$ при отображении (41), $\mu_{w}$ - равновесная мера множества $E_{\zeta}$ при наличии внешнего поля (веса) $w(\zeta)=$ $1 / \sqrt{\zeta^{2}+1}$. Но непосредственно из определения равновесной меры следует, что $\mu_{w}\left(E_{j}^{(\zeta)}\right)=\mu\left(\Gamma_{j}\right)$, где $\mu$ - обычная равновесная мера множества $\Gamma_{E}$, что, в свою очередь, по определению гармонической меры равно $\omega_{j}(\infty)$. Таким образом, лемма доказана для системы дуг, на которой сушествует комплексньй $T$-многочлен, причем из (40) и (37)

$$
\omega_{j+1}(\infty)=\frac{m_{j}^{(N)}}{N}, \quad j=1, \ldots, l-1
$$

Для завершения доказательства леммы достаточно воспользоваться тем фактом, что рассмотренные системы дуг всюду плотны в множестве произвольных систем дуг $\Gamma_{E}$ (он был установлен для систем отрезков действительной оси различньми способами в [25], [33], [34]).

\section{§4. Доказательство теоремы 1}

а) Нетрудно видеть (см. также [20]), что псевдопериодичность последовательности круговых параметров $a_{n}=-\overline{P_{n+1}(0)}$ равносильна периодичности последовательности круговых параметров $\widetilde{a}_{n}$ многочленов

$$
\widetilde{P}_{n}(z)=\exp (-\mathrm{i} n \theta) \cdot P_{n}(\exp (\mathrm{i} \theta) z)
$$

Согласно [20; теорема 4.4] периодичность последовательности круговых параметров $\left\{a_{n}\right\},\left|a_{n}\right|<1$, начиная с некоторого номера $n_{0}$ равносильна ортогональности последовательности многочленов $P_{n+1}(z)=z P_{n}(z)-\bar{a}_{n} P_{n}^{*}(z), P_{0}(z)=1$, относительно некоторой меры $d \sigma(\varphi ; A, W, \lambda)$ и сушествованию на соответствуюшем $\Gamma_{E}$ комплексного $T$-многочлена, т.е. такого самовзаимного многочлена

$$
\mathscr{T}_{N}(z)=e^{\mathrm{i} \psi} z^{N}+\cdots
$$

что для некоторого самовзаимного многочлена $\mathscr{U}_{N-l}$ справедливо равенство (38). Но тогда (43) доказывает требуемое. С другой стороны, из (43) и леммы 6 следует $N$-периодичность ( начиная с некоторого номера) последовательности $\left\{b_{j}^{(n)}\right\}$ и равенство $k_{j}^{(n+N)}=k_{j}^{(n)}+m_{j}^{(N)}$ в системе $(28)$ для любой меры $d \sigma(\varphi ; A, W, \lambda)$ с соответствующим множеством $E$. Тогда псевдопериодичность последовательности круговых параметров непосредственно следует из лемм 1,4 и 5 .

б) Доказательство теоремы 1, б) будет состоять из нескольких шагов. 
На первом шаге установим, что точки $b_{j}^{(n)}, j=1, \ldots, l-1$, образуют всюду плотное множество в наборе $b_{j}^{0}(x), j=1, \ldots, l-1$, параметризованном переменньгми $x \in \mathbb{R}^{l-1}$ (см. (50) далее). Для этого используем аппроксимационную теорему Кронекера (см., например, [35]): если числа $\omega_{2}(\infty), \ldots, \omega_{l}(\infty), 1$ независимы над полем рациональных чисел, то для любых заданных действительных чисел $y_{1}, \ldots, y_{l-1}$ и любой последовательности $\widetilde{\varepsilon}_{k} \downarrow 0$ сушествует возрастаюшая последовательность натуральных чисел $\left\{q_{k}\right\}$ и $l-1$ последовательностей целых чисел $\widetilde{m}_{k, p}, p=1, \ldots, l-1$, таких, что

$$
q_{k} \omega_{j+1}(\infty)=y_{j}+\widetilde{m}_{k, j}+\widetilde{\varepsilon}_{k, j},
$$

где $\left|\widetilde{\varepsilon}_{k, j}\right|<\widetilde{\varepsilon}_{k}, j=1, \ldots, l-1, k=1, \ldots$ Условие теоремы Кронекера здесь вьполнено, поскольку $\omega_{1}(\infty)+\cdots+\omega_{l}(\infty)=1$. Так как матрица $A=\left(a_{i j}\right)$ является матрицей периодов римановой поверхности $\mathscr{S}$ (см. [27]), то она невырождена, поэтому, умножая (44) на $A$, получим

$$
\sum_{j=1}^{l-1} a_{j p} \omega_{j+1}(\infty) q_{k}=\sum_{j=1}^{l-1} a_{j p} y_{j}+\sum_{j=1}^{l-1} a_{j p} \widetilde{m}_{k, j}+\sum_{j=1}^{l-1} a_{j p} \widetilde{\varepsilon}_{k, j}, \quad p=1, \ldots, l-1 .
$$

При этом невырожденность и действительность $A$ позволяет утверждать, что $\sum_{j=1}^{l-1} a_{j p} y_{j}, p=1, \ldots, l-1$, могут быть любыми действительными числами. С учетом леммы 6 отсюда следует, что для любых действительных чисел $x_{1}, \ldots, x_{l-1}$ и любой последовательности $\varepsilon_{k} \downarrow 0$ сушествует возрастаюшая последовательность натуральных чисел $\left\{q_{k}\right\}$ и $l-1$ последовательностей целых чисел $\widetilde{m}_{k, p}, p=1, \ldots, l-1$, таких, что

$$
2 q_{k} \operatorname{Re} \Phi_{p}(\xi)=\sum_{j=1}^{l-1} \widetilde{m}_{k, j} a_{j p}+x_{p}+\varepsilon_{k, p}
$$

где $\left|\varepsilon_{k, p}\right|<\varepsilon_{k}, p=1, \ldots, l-1$.

Выбрав такие последовательности, положим в основной системе (28) $n=q_{k}$ и $n=q_{k}+1$. Получим

$$
\begin{gathered}
\exp \left(\left(-a-\frac{l}{2}+w+1\right) \Phi_{j}(\xi)-\left(-a-\frac{l}{2}+w\right) \Phi_{j}(\bar{\xi})+\sum_{i=1}^{m} \lambda_{i} m_{i} \Phi_{j}\left(v_{i}\right)\right. \\
\left.-\sum_{i=1}^{l-1} \Phi_{j}\left(b_{i}^{\left(q_{k}\right)}\right)+\sum_{i=1}^{2 w} \Phi_{j}\left(u_{i}\right)-\sum_{i=1}^{l-1}\left(k_{i}^{\left(q_{k}\right)}-\widetilde{m}_{k, i}\right) a_{j i}+\varepsilon_{k, j}+x_{j}\right)=1 \\
j=1, \ldots, l-1 \\
\exp \left(\left(2-a-\frac{l}{2}+w\right) \Phi_{j}(\xi)-\left(1-a-\frac{l}{2}+w\right) \Phi_{j}(\bar{\xi})+\sum_{i=1}^{m} \lambda_{i} m_{i} \Phi_{j}\left(v_{i}\right)+x_{j}\right. \\
\left.-\sum_{i=1}^{l-1} \Phi_{j}\left(b_{i}^{\left(q_{k}+1\right)}\right)+\sum_{i=1}^{2 w} \Phi_{j}\left(u_{i}\right)-\sum_{i=1}^{l-1}\left(k_{i}^{\left(q_{k}+1\right)}-\widetilde{m}_{k, i}\right) a_{j i}+\varepsilon_{k, j}\right)=1 \\
j=1, \ldots, l-1
\end{gathered}
$$


Отсюда

$$
\begin{aligned}
\exp & \left(\Phi_{p}(\xi)-\Phi_{p}(\bar{\xi})-\sum_{j=1}^{l-1}\left(\Phi_{p}\left(b_{j}^{\left(q_{k}+1\right)}\right)-\Phi_{p}\left(b_{j}^{\left(q_{k}\right)}\right)\right)\right. \\
& \left.-\sum_{j=1}^{l-1}\left(k_{j}^{\left(q_{k}+1\right)}-k_{j}^{\left(q_{k}\right)}\right) a_{j p}\right)=1, \quad p=1, \ldots, l-1 .
\end{aligned}
$$

Тогда, учитывая ограниченность $\Phi_{j}, j=1, \ldots, l-1$, на фундаментальной области $\mathbb{T}$, можно найти подпоследовательность из $\left\{q_{k}\right\}$ (мы сохраним для нее прежнее обозначение) такую, что

$$
b_{j}^{\left(q_{k}\right)} \rightarrow b_{j}^{0}(x), \quad b_{j}^{\left(q_{k}+1\right)} \rightarrow b_{j}^{1}(x), \quad k_{j}^{\left(q_{k}+1\right)}-k_{j}^{\left(q_{k}\right)} \rightarrow k_{j}(x) \text { при } k \rightarrow \infty,
$$

а подставляя (49) в (46), видим, что

$$
\widetilde{m}_{k, i}-k_{i}^{\left(q_{k}\right)} \rightarrow \widetilde{m}_{i}(x), \quad k \rightarrow \infty .
$$

При этом (46) и (48) дадут в пределе соответственно

$$
\begin{gathered}
\exp \left(\left(w-a-\frac{l}{2}+1\right) \Phi_{j}(\xi)-\left(w-a-\frac{l}{2}\right) \Phi_{j}(\bar{\xi})-\sum_{i=1}^{l-1} \Phi_{j}\left(b_{i}^{0}(x)\right)\right. \\
\left.+\sum_{i=1}^{m} \lambda_{i} m_{i} \Phi_{j}\left(v_{i}\right)+\sum_{i=1}^{2 w} \Phi_{j}\left(u_{i}\right)+x_{j}+\sum_{i=1}^{l-1} \tilde{m}_{i}(x) a_{j i}\right)=1 \\
j=1, \ldots, l-1, \\
\exp \left(\left(\Phi_{j}(\xi)-\Phi_{j}(\bar{\xi})\right)-\sum_{i=1}^{l-1}\left(\Phi_{j}\left(b_{i}^{1}(x)\right)-\Phi_{j}\left(b_{i}^{0}(x)\right)\right)-\sum_{i=1}^{l-1} k_{i}(x) a_{j i}\right)=1 \\
j=1, \ldots, l-1
\end{gathered}
$$

Соотношения (49) и (50) означают, что точки $\left(b_{1}^{(n)}, \ldots, b_{l-1}^{(n)}\right)$ образуют всюду плотное множество в наборе $\left\{\left(b_{1}^{0}(x), \ldots, b_{l-1}^{0}(x)\right), x \in \mathbb{R}^{l-1}\right\}$.

На втором шаге установим аналитичность по $x$ на $\mathbb{R}^{l-1}$ функций $\phi\left(b_{j}^{p}(x)\right), j=$ $1, \ldots, l-1, p=0,1$. Так как $\Phi_{j}$ являются не чем иным, как абелевыми интегралами первого рода [28; с. 66] на римановой поверхности $\mathscr{S}$, то равенства $(50)$ являются переформулировкой проблемы обрашения Якоби на $\mathscr{S}$. Как известно (см., например, [36; лекция 10]), отвечающее ей отображение Якоби бианалитично в каждой точке, в которой проблема обрашения Якоби имеет единственное решение.

Хорошо известно, что единственность решения проблемы обрашения Якоби нарушается лишш в точках, образующих специальный дивизор на поверхности $\mathscr{S}$. Поскольку поверхность $\mathscr{S}$ гиперэллиптическая, то нетрудно установить (см. также [36; лекция 9], [37; лемма А.20], [38]), что дивизор на поверхности $\mathscr{S}$, соответствующий точкам $b_{1}^{0}(x), \ldots, b_{l-1}^{0}(x)$, среди которых нет совпадающих, специален, если и только если среди точек $\phi\left(b_{1}^{0}(x)\right), \ldots, \phi\left(b_{l-1}^{0}(x)\right)$ есть совпадающие. В общем случае для специальности такого дивизора $\mathscr{D}$ необходимо и достаточно, чтобы 
степень дивизора $\widetilde{\mathscr{D}}$ на $\mathbb{C}$, в который каждая из точек $\phi\left(b_{i}^{0}(x)\right)$ входит с кратностью, равной максимальной из кратностей точек $b_{i}^{0}(x)$ и $-b_{i}^{0}(x)$ дивизора $\mathscr{D}$, была меньше $l-1, \operatorname{deg}(\widetilde{\mathscr{D}})<l-1$.

Заметим, что если дивизор, соответствуюший точкам $\beta_{1}, \ldots, \beta_{l-1}$, совпадает с дивизором, задаваемым точками $\widetilde{b}_{1}^{(n)}, \ldots, \widetilde{b}_{l-1}^{(n)}$, отвечающим какому-нибудь весу $d \sigma(\varphi ; \widetilde{A}, \widetilde{W}, \widetilde{\lambda})$ с тем же $R$, то он неспециален. В самом деле, каждое решение системы (28) соответствует по лемме 4 ортогональному многочлену степени $n$ относительно веса $d \sigma(\varphi ; \widetilde{A}, \widetilde{W}, \widetilde{\lambda})$. Так как такой ортогональный многочлен единствен, то и решение системы (28), рассматриваемой как проблема обрашения Якоби, единственно, что и дает требуемое.

Теперь, чтобы установить неспециальность дивизора, соответствуюшего точкам $b_{1}^{0}(x), \ldots, b_{l-1}^{0}(x)$, достаточно доказать, что они совпадут с $\widetilde{b}_{1}^{(n)}, \ldots, \widetilde{b}_{l-1}^{(n)}$, отвечающими какому-нибудь весу $d \sigma(\varphi ; \widetilde{A}, \widetilde{W}, \widetilde{\lambda})$ с тем же $R$. Для этого посмотрим на (50) как на проблему обращения Якоби относительно $l-1$ "неизвестных" различных точек из $v_{j}, \phi\left(v_{j}\right) \in \Gamma \backslash \Gamma_{E}$, и $u_{j}$. (То, что имеется по крайней мере $l-1$ такая точка, легко следует из (3) после перехода от алгебраических многочленов $A$ и $W$ к их тригонометрическим аналогам как в [19].) Так как эта проблема обрашения Якоби имеет единственное решение, то она имеет единственное решение и в некоторой окрестности точек $x_{j}, j=1, \ldots, l-1$, в $\mathbb{R}^{l-1}$. В частности, можно взять $x_{j}+\varepsilon_{k, j}$ для достаточно больших $k$, т.е. сушествует единственное решение системы

$$
\begin{aligned}
& \exp \left(\left(w-a-\frac{l}{2}+1\right) \Phi_{j}(\xi)-\left(w-a-\frac{l}{2}\right) \Phi_{j}(\bar{\xi})-\sum_{i=1}^{l-1} \Phi_{j}\left(b_{i}^{0}(x)\right)\right. \\
& \quad+\sum_{i=1}^{m-l+1} \lambda_{i} m_{i} \Phi_{j}\left(v_{i}\right)+\sum_{i=1}^{2 w} \Phi_{j}\left(u_{i}\right)+x_{j}+\varepsilon_{k, j}-\sum_{i=1}^{l-1}\left(k_{i}^{\left(q_{k}\right)}-\widetilde{m}_{k, i}\right) a_{j i} \\
& \left.\quad+\sum_{i=m-l+2}^{m} \lambda_{i} \Phi_{j}\left(\widetilde{v}_{i}\right)\right)=1, \quad j=1, \ldots, l-1,
\end{aligned}
$$

рассматриваемой как система уравнений относительно $\widetilde{v}_{i}, i=1, \ldots, l-1$. Здесь мы считаем для простоты этими $l-1$ различными точками последние из нулей $A(\phi(u))$, т.е. $v_{m-l+2}, \ldots, v_{m}$, причем кратности каждой из них пусть равны 1 . Если этих точек не хватит (скажем, при $m=l-2$ ), то нужно добавить соответствующее количество точек $u_{j}$, т.е. вместо $\sum_{i=1}^{2 w} \Phi_{j}\left(u_{i}\right)$ взять $\sum_{i=1}^{2 w-1} \Phi_{j}\left(u_{i}\right) \pm \Phi_{j}\left(\widetilde{v}_{l-1}\right)$, тогда $2 \widetilde{w}=2 w-1$. Подставляя в (52) равенства (45), получаем

$$
\begin{aligned}
& \exp \left(\left(2 q_{k}+w-a-\frac{l}{2}+1\right) \Phi_{j}(\xi)-\left(2 q_{k}+w-a-\frac{l}{2}\right) \Phi_{j}(\bar{\xi})\right. \\
& -\sum_{i=1}^{l-1} \Phi_{j}\left(b_{i}^{0}(x)\right)+\sum_{i=1}^{m-l+1} \lambda_{i} m_{i} \Phi_{j}\left(v_{i}\right)+\sum_{i=1}^{2 w} \Phi_{j}\left(u_{i}\right) \\
& \left.-\sum_{i=1}^{l-1} k_{i}^{\left(q_{k}\right)} a_{j i}+\sum_{i=m-l+2}^{m} \lambda_{i} \Phi_{j}\left(\widetilde{v}_{i}\right)\right)=1, \quad j=1, \ldots, l-1 .
\end{aligned}
$$


Равенства (53) совпадают с системой (28), в которой изменен полином $A$, т.е. $\widetilde{A}(z)=$ $\widetilde{c}_{A} \prod_{j=1}^{m-l+1}\left(z-z_{j}\right)^{m_{j}} \prod_{j=m-l+2}^{m}\left(z-\widetilde{z}_{j}\right)$, где $\widetilde{z}_{j}=\phi\left(\widetilde{v}_{j}\right), j=m-l+2, \ldots, m$. Для $2 \widetilde{w}=2 w-\kappa$ изменятся и $m$, и $W(\widetilde{W}(z)$ будет являться делителем $W)$, кроме того, $\widetilde{\lambda}_{j}$ будут выбраны согласно тому, на каком листе находится точка $\widetilde{v}_{j}$. Это означает по лемме 4 , что точки $b_{1}^{0}(x), \ldots, b_{l-1}^{0}(x)$ совпадают с точками $\widetilde{b}_{1}^{\left(q_{k}\right)}, \ldots, \widetilde{b}_{l-1}^{\left(q_{k}\right)}$, отвечающими весу $d \sigma(\varphi ; \widetilde{A}, \widetilde{W}, \widetilde{\lambda})$.

Таким образом, дивизор, соответствуюший точкам $b_{1}^{0}(x), \ldots, b_{l-1}^{0}(x)$, неспециален для любого $x \in \mathbb{R}^{l-1}$.

Перейдем к завершаюшему этапу. Так как

$$
\begin{aligned}
& \lim _{k \rightarrow \infty} \overline{\left(\frac{a_{q_{k}+1}}{a_{q_{k}}}\right)}=C_{\phi} \frac{\overline{\Omega(\xi,-\xi)}}{\Omega(\xi,-\xi)} \prod_{j=1}^{l-1} \frac{\Omega\left(\xi, b_{j}^{0}(x)\right) \Omega\left(-\bar{\xi}, b_{j}^{1}(x)\right)}{\Omega\left(-\bar{\xi}, b_{j}^{0}(x)\right) \Omega\left(\xi, b_{j}^{1}(x)\right)} \\
& \quad \times \exp \sum_{j=1}^{l-1} k_{j}(x)\left(\Phi_{j}(-\bar{\xi})-\Phi_{j}(\xi)\right),
\end{aligned}
$$

то множество предельных точек последовательности $\left(\overline{a_{n+1} / a_{n}}\right)$ содержит множество значений функции

$$
\begin{aligned}
F(x) \stackrel{\text { def }}{=} & C_{\phi} \frac{\overline{\Omega(\xi,-\xi)}}{\Omega(\xi,-\xi)} \prod_{j=1}^{l-1} \frac{\Omega\left(\xi, b_{j}^{0}(x)\right) \Omega\left(-\bar{\xi}, b_{j}^{1}(x)\right)}{\Omega\left(-\bar{\xi}, b_{j}^{0}(x)\right) \Omega\left(\xi, b_{j}^{1}(x)\right)} \\
& \times \exp \sum_{j=1}^{l-1} k_{j}(x)\left(\Phi_{j}(-\bar{\xi})-\Phi_{j}(\xi)\right) .
\end{aligned}
$$

С другой стороны, какую бы предельную точку последовательности $\overline{a_{n+1} / a_{n}}$ мы ни взяли, скажем $D=\lim _{k \rightarrow \infty} \overline{a_{n_{k}} / a_{n_{k}-1}}$, по лемме 5 имеем

$$
\begin{aligned}
D= & C_{\phi} \lim _{k \rightarrow \infty} \frac{\overline{\Omega(\xi,-\xi)}}{\Omega(\xi,-\xi)} \prod_{j=1}^{l-1} \frac{\Omega\left(\xi, b_{j}^{\left(n_{k}\right)}\right) \Omega\left(-\bar{\xi}, b_{j}^{\left(n_{k}+1\right)}\right)}{\Omega\left(-\bar{\xi}, b_{j}^{\left(n_{k}\right)}\right) \Omega\left(\xi, b_{j}^{\left(n_{k}+1\right)}\right)} \\
& \times \exp \sum_{j=1}^{l-1}\left(k_{j}^{\left(n_{k}+1\right)}-k_{j}^{\left(n_{k}\right)}\right)\left(\Phi_{j}(-\bar{\xi})-\Phi_{j}(\xi)\right),
\end{aligned}
$$

причем $b_{j}^{\left(n_{k}\right)}$ и $b_{j}^{\left(n_{k}+1\right)}$ удовлетворяют основной системе уравнений (28). Следовательно, справедливы равенства (48), где $q_{k}=n_{k}$. Выбирая из последовательностей $b_{j}^{\left(n_{k}\right)}$ и $b_{j}^{\left(n_{k}+1\right)}$ подпоследовательности, сходящиеся к точкам $b_{j, 0}$ и $b_{j, 1} \in \mathbb{T}$ соответственно (для полученной подпоследовательности тогда будут существовать пределы $\left.\lim _{i \rightarrow \infty}\left(k_{j}^{\left(n_{i}+1\right)}-k_{j}^{\left(n_{i}\right)}\right)=k_{j} \in \mathbb{Z}\right)$, найдем, записывая равенства $(28)$ для этих подпоследовательностей и переходя в них к пределу, что существуют пределы

$$
\begin{gathered}
\lim _{i \rightarrow \infty} \exp \left(2 n_{i} \operatorname{Re} \Phi_{p}(\xi)-\sum_{j=1}^{l-1} k_{j}^{\left(n_{i}\right)} a_{j p}\right)=\exp \kappa_{p} \stackrel{\text { def }}{=} \exp \left(\left(a+\frac{l}{2}-w-1\right) \Phi_{p}(\xi)\right. \\
\left.-\left(a+\frac{l}{2}-w\right) \Phi_{p}(\bar{\xi})-\sum_{j=1}^{m} \lambda_{j} m_{j} \Phi_{p}\left(v_{j}\right)-\sum_{i=1}^{2 w} \Phi_{j}\left(u_{i}\right)+\sum_{j=1}^{l-1} \Phi_{p}\left(b_{j, 0}\right)\right) \\
p=1, \ldots, l-1,
\end{gathered}
$$




$$
\begin{aligned}
& \lim _{i \rightarrow \infty} \exp \left(2\left(n_{i}+1\right) \operatorname{Re} \Phi_{p}(\xi)-\sum_{j=1}^{l-1} k_{j}^{\left(n_{i}+1\right)} a_{j p}\right) \\
& \quad=\exp \left(\kappa_{p}+\sum_{j=1}^{l-1}\left(\Phi_{p}\left(b_{j, 1}\right)-\Phi_{p}\left(b_{j, 0}\right)\right)\right), \quad p=1, \ldots, l-1 .
\end{aligned}
$$

Учитьвая отмеченную вьше единственность решения системы уравнений (50), получаем, что $b_{j, 0}=b_{j}^{0}(\kappa), b_{j, 1}=b_{j}^{1}(\kappa), \kappa=\left(\kappa_{1}, \ldots, \kappa_{l-1}\right)$, откуда вытекает, что множество предельных точек последовательности $\left(\overline{a_{n+1} / a_{n}}\right)$ содержится во множестве значений функции $F(x), x \in \mathbb{R}^{l-1}$, а значит, совпадает с ним.

Заметим также, что приведенное рассуждение применимо и к доказательству того, что множество значений функции $F(x), x \in \mathbb{R}^{l-1}$, содержит $\left\{\overline{a_{n+1} / a_{n}}: n \geqslant\right.$ $a+l / 2+v\}$.

Таким образом, для того чтобы установить требуемоеповедение последовательности $\left(a_{n+1} / a_{n}\right)$, достаточно убедиться в том, что $F(x)$ - непостоянная аналитическая функция на $\mathbb{R}^{l-1}$.

Прежде всего заметим, что функции $\phi\left(b_{j}^{s}(x)\right), j=1, \ldots, l-1, s=0,1$, аналитические на $\mathbb{R}^{l-1}$ (для $s=0$ это было установлено ранее, для $s=1$ доказывается аналогично). Сами функции $b_{j}^{0}(x)$ и $b_{j}^{1}(x)$ лишш кусочно аналитичны, так как терпят разрыв при $b_{j}^{s}(x) \in \partial K_{p}$ (переходу на другой лист соответствует переход через $\partial K_{p}$ и $\partial K_{p}^{\prime}$ и изменение $\left.k_{j}(x)\right)$. Проследим подробнее за изменением $F(x)$ при таком переходе.

Пусть, например, $b_{i}^{0}(x)$ при $i \neq j$ и $b_{i}^{1}(x)$ при $i=1, \ldots, l-1$ аналитические в $x^{(0)}, \mathrm{a}$

$$
\lim _{n \rightarrow \infty} b_{j}^{0}\left(x^{(n, 1)}\right)=b_{j}^{0}\left(x^{(0)}\right), \quad \lim _{n \rightarrow \infty} b_{j}^{0}\left(x^{(n, 2)}\right)=T_{p}\left(b_{j}^{0}\left(x^{(0)}\right)\right), \quad \lim _{n \rightarrow \infty} x^{(n, s)}=x^{(0)},
$$

$s=1,2$. Тогда из равенств $(51)$, полагая в них $x=x^{(n, 2)}$ и переходя к пределу при $n \rightarrow \infty$, получаем с учетом (10), что

$$
\begin{gathered}
\exp \left(\left(\Phi_{q}(\xi)-\Phi_{q}(\bar{\xi})\right)-\sum_{i=1}^{l-1}\left(\Phi_{q}\left(b_{i}^{1}\left(x^{(0)}\right)\right)-\Phi_{q}\left(b_{i}^{0}\left(x^{(0)}\right)\right)\right)+a_{q p}\right. \\
\left.-\sum_{i=1}^{l-1} \lim _{n \rightarrow \infty} k_{i}\left(x^{(n, 2)}\right) a_{q i}\right)=1, \quad q=1, \ldots, l-1 .
\end{gathered}
$$

Сравнивая эти равенства с (51) при $x=x^{(0)}$, имеем $k_{i}\left(x^{(n, 2)}\right)=k_{i}\left(x^{(0)}\right), i \neq p$, и $k_{p}\left(x^{(n, 2)}\right)=k_{p}\left(x^{(0)}\right)+1$ для достаточно больших $n$. Следовательно, пользуясь формулами $(9),(10)$, получим, что для всех $x$ из некоторой окрестности точки $x^{(0)}$ функция $F(x)$ может быть записана следующим образом:

$$
\begin{aligned}
F(x)= & C_{\phi} \frac{\overline{\Omega(\xi,-\xi)}}{\Omega(\xi,-\xi)} \cdot \frac{\Omega\left(\xi, \widetilde{b}_{j}^{0}(x)\right)}{\Omega\left(-\bar{\xi}, \widetilde{b}_{j}^{0}(x)\right)} \prod_{\substack{i=1 \\
i \neq j}}^{l-1} \frac{\Omega\left(\xi, b_{i}^{0}(x)\right)}{\Omega\left(-\bar{\xi}, b_{i}^{0}(x)\right)} \\
& \times \prod_{i=1}^{l-1} \frac{\Omega\left(-\bar{\xi}, b_{i}^{1}(x)\right)}{\Omega\left(\xi, b_{i}^{1}(x)\right)} \exp \sum_{i=1}^{l-1} k_{i}\left(x^{(0)}\right)\left(\Phi_{i}(-\bar{\xi})-\Phi_{i}(\xi)\right),
\end{aligned}
$$


где

$$
\widetilde{b}_{j}^{0}(x)= \begin{cases}b_{j}^{0}(x) & \text { при таких } x \in \mathbb{R}^{l-1}, \text { что } b_{j}^{0}(x) \in \mathscr{U}\left(b_{j}^{0}\left(x^{(0)}\right)\right) \cap \mathbb{T}, \\ T_{p}^{-1}\left(b_{j}^{0}(x)\right) & \text { при таких } x \in \mathbb{R}^{l-1}, \text { что } b_{j}^{0}(x) \in \mathscr{U}\left(T_{p}\left(b_{j}^{0}\left(x^{(0)}\right)\right)\right) \cap \mathbb{T}\end{cases}
$$

для подходящим образом выбранных окрестностей $\mathscr{U}\left(b_{j}^{0}\left(x^{(0)}\right)\right)$ и $\mathscr{U}\left(T_{p}\left(b_{j}^{0}\left(x^{(0)}\right)\right)\right)$. Поскольку $\phi\left(\widetilde{b}_{j}^{0}(x)\right)$ аналитична в выбранной окрестности точки $x^{(0)}$ и $\phi$ осушествляет конформное отображение окрестности точки $u=b_{j}^{0}\left(x^{(0)}\right)$ в плоскости $u$ на окрестность точки $\phi\left(b_{j}^{0}\left(x^{(0)}\right)\right)$, функция $\widetilde{b}_{j}^{0}(x)$ аналитична в окрестности точки $x^{(0)}$.

Таким образом, функция $F(x)$ аналитическая на $\mathbb{R}^{l-1}$.

Установим теперь, что она не может быть постоянной на $\mathbb{R}^{l-1}$.

Выберем какое-нибудь значение $x_{0}$ так, чтобы $b_{j}^{0}\left(x_{0}\right) \neq \bar{\xi}, \quad b_{j}^{1}\left(x_{0}\right) \neq \xi, j=$ $1, \ldots, l-1$. Это можно сделать в силу того, что множество значений функции $F(x)$, $x \in \mathbb{R}^{l-1}$, содержит множество $\left\{\overline{a_{n+1} / a_{n}}: n \geqslant a+l / 2+v\right\}$, а равенство $a_{n}=0$ при всех $n \geqslant a+l / 2+v$ невозможно, поскольку вес $d \sigma$ не совпадает с весом Бернштейна-Сегё.

Функции $b_{1}^{0}(x)$ и $b_{1}^{1}(x)$ продолжимы аналитически в некоторую окрестность $U\left(x_{0}\right) \subset \mathbb{C}^{l-1}$. Так как они непостоянны, то можно считать, что $b_{j}^{0}\left(x_{0}\right) \neq \pm \bar{\xi}$, $b_{j}^{1}\left(x_{0}\right) \neq \pm \xi, j=1, \ldots, l-1$. Обозначим через $b_{T}^{s}, s=0,1$, дивизоры, компоненты которых таковы:

$$
b_{1, T}^{0}=\bar{\xi}, \quad b_{1, T}^{1}=\xi, \quad b_{j, T}^{s}=b_{j}^{s}\left(x_{0}\right), \quad j=2, \ldots, l-1, \quad s=0,1 .
$$

Эти дивизоры неспециальные. Соединим теперь $b_{1}^{s}\left(x_{0}\right)$ с $b_{1, T}^{s}, s=0,1$, аналитическими кривьми в $\mathbb{T}$, не проходящими через точки $\pm b_{j, T}^{s}, j=1,2, \ldots, l-1$. Таким образом, построены аналитические функции $\chi_{s}(t), s=0,1$, параметра $t \in[0,1]$ такие, что $\chi_{s}(0)=b_{1}^{s}\left(x_{0}\right), \chi_{s}(1)=b_{1, T}^{s}, s=0,1$. Им отвечают в силу неспециальности дивизоров $\left(\chi_{s}(t), b_{2, T}^{s}, \ldots, b_{l-1, T}^{1}\right)$ аналитические кривые

$$
\begin{aligned}
x_{p, s}(t)= & \left(a+\frac{l}{2}-w-1\right) \Phi_{p}(\xi)+\left(w-a-\frac{l}{2}\right) \Phi_{p}(\bar{\xi})-\sum_{j=1}^{m} \lambda_{j} m_{j} \Phi_{p}\left(v_{j}\right) \\
& -\sum_{i=1}^{2 w} \Phi_{p}\left(u_{i}\right)+\sum_{j=2}^{l-1} \Phi_{p}\left(b_{j, T}^{s}\right)+\Phi_{p}\left(\chi_{s}(t)\right)+\sum_{i=1}^{l-1} \widetilde{m}_{i}\left(x_{0}\right) a_{p i}
\end{aligned}
$$

в $\mathbb{C}, p=1, \ldots, l-1, s=0,1$.

Итак, предположение о постоянстве функции $F(x)$ на $\mathbb{R}^{l-1}$ приводит к выводу о постоянстве аналитической функции параметра $t$

$$
\begin{aligned}
\widetilde{F}(t)= & C_{\phi} \frac{\Omega\left(\xi, \chi_{0}(t)\right) \Omega\left(-\bar{\xi}, \chi_{1}(t)\right)}{\Omega\left(-\bar{\xi}, \chi_{0}(t)\right) \Omega\left(\xi, \chi_{1}(t)\right)} \cdot \frac{\overline{\Omega(\xi,-\xi)}}{\Omega(\xi,-x \mathrm{i})} \\
& \times \prod_{j=2}^{l-1} \frac{\Omega\left(\xi, b_{j, T}^{0}\right) \Omega\left(-\bar{\xi}, b_{j, T}^{1}\right)}{\Omega\left(-\bar{\xi}, b_{j, T}^{0}\right) \Omega\left(\xi, b_{j, T}^{1}\right)} \exp \sum_{j=1}^{l-1} k_{j}\left(x_{0}\right)\left(\Phi_{j}(-\bar{\xi})-\Phi_{j}(\xi)\right)
\end{aligned}
$$


в некоторой окрестности нуля, а следовательно, и на всем отрезке [0, 1]. Отсюда вытекает, что предполагаемое постоянное значение $F(x)$ совпадает с $\widetilde{F}(1)=\infty$, что, как отмечено выше, невозможно.

Аналогично рассматривается последовательность $\left(a_{n+k} / a_{n}\right)_{n=1}^{\infty}$ для любого $k \in \mathbb{N}$.

Перейдем к доказательству утверждения о последовательности модулей круговых параметров. Так как по лемме 5 с учетом леммы 1

$$
\left|a_{n-1}\right|=\prod_{j=1}^{l-1}\left|\frac{\Omega\left(-\bar{\xi}, b_{j}^{(n)}\right)}{\Omega\left(\xi, b_{j}^{(n)}\right)}\right| \cdot\left|\frac{\Omega(\xi, \bar{\xi})}{\Omega(-\bar{\xi}, \bar{\xi})}\right|,
$$

то, рассуждая как и выше, убедимся, что все предельные точки последовательности $\left\{\left|a_{n}\right|\right\}$ имеют вид

$$
F_{1}(x) \stackrel{\text { def }}{=} \prod_{j=1}^{l-1}\left|\frac{\Omega\left(-\bar{\xi}, b_{j}(x)\right)}{\Omega\left(\xi, b_{j}(x)\right)}\right| \cdot\left|\frac{\Omega(\xi, \bar{\xi})}{\Omega(-\bar{\xi}, \bar{\xi})}\right|,
$$

где $x=\left(x_{1}, \ldots, x_{l-1}\right)$ пробегает множество $\mathbb{R}^{l-1}$. Аналитичность функции

$$
\prod_{j=1}^{l-1} \frac{\Omega\left(-\bar{\xi}, b_{j}(x)\right)}{\Omega\left(\xi, b_{j}(x)\right)} \exp \sum_{j=1}^{l-1} \widetilde{m}_{j}(x) \cdot\left(\Phi_{j}(\xi)-\Phi_{j}(\bar{\xi})\right)
$$

на $\mathbb{R}^{l-1}$ устанавливается аналогично предыдушему. Так как ее модуль совпадает с $F_{1}(x)$, то теорема будет доказана, как только мы установим непостоянство $F_{1}(x)$ на $\mathbb{R}^{l-1}$. Но по доказанному постоянство $F_{1}(x)$ означало бы, что для достаточно больших $n$

$$
\left|a_{n}\right|=K, \quad 0 \leqslant K \leqslant 1 .
$$

Покажем, что это невозможно. Для этого используем установленное в [29] равенство

$$
\left\|P_{n}\right\|_{L^{2}\left(\Gamma_{E}\right)}^{2}=c \prod_{k=0}^{n-1}\left(1-\left|a_{k}\right|^{2}\right) .
$$

Тогда равенства (56), (57) противоречат теореме Видома (см. [12; теорема 9.2 и п. 12]), гласящей, что при предположениях теоремы 1,б) множество предельных точек последовательности $\left\|P_{n}\right\|_{L^{2}\left(\Gamma_{E}\right)}^{2}\left(C\left(\Gamma_{E}\right)\right)^{2 n}$ есть невырожденный промежуток.

Полученное противоречие означает, что множество предельных точек последовательности $\left\{\left|a_{n}\right|\right\}$ является некоторьм отрезком в $[0,1]$.

ЗАмЕчАниЕ 2. В работе [17] установлено, что предположение о квазипериодичности и даже о почти периодичности бибесконечной последовательности круговых параметров $\left\{a_{n}\right\}$ равносильно тому, что мера ортогональности имеет вид, являющийся частньм случаем рассмотренного в настоящей работе. Отсюда можно было бы попытаться получить квазипериодичность рассматриваемых последовательностей с помощью техники конечномерных возмушений (см., например, обзор [39]), но для того, чтобы доказать утверждение теоремы, необходимо так же, как и вьше, установить непостоянство соответствующих функций и другие их свойства. 
ЗАмечание 3. Основные результаты статьи были анонсированы в [40], [41].

ЗАмечание 4. Используя [10; лемма] и перенося методы работ [14; теорема 4], [31], [42] с нескольких отрезков действительной оси на несколько дуг единичной окружности, можно доказать справедливость утверждения теоремы $1, б)$ и для весов вида $|W t / \sqrt{R}|$, где $t$ - положительная на $\Gamma_{E}$ функция с непрерывной второй производной.

ЗАмечАниЕ 5. Другое доказательство теоремы 1 для $l=2$ (с использованием аппарата эллиптических функций) дано в [43].

\section{Список литературы}

1. Сегё Г. Ортогональные многочлены. М.: ГИФМЛ, 1962.

2. Геронимус $Я$.Л. Многочлены, ортогональные на окружности и на отрезке (оценки, асимптотические формулы, ортогональные ряды). М.: ГИФМЛ, 1958.

3. Jones W.B., Njåstad O., Thron W.J. Moment theory, orthogonal polynomials, quadrature, and continued fractions associated with the unit circle // Bull. London Math. Soc. 1989. V. 21. P. $113-152$.

4. Рахманов E. A. Об асимптотических свойствах многочленов, ортогональных на окружности с весами, не удовлетворяющими условию Сегё // Матем. сб. 1986. Т. 130 (172). C. $151-169$.

5. Бадков В. М. Асимптотические и экстремальные свойства полиномов при наличии особенностей у веса // Труды МИАН. 1992. Т. 198. С. 41-88.

6. Суетин С. П. Аппроксимации Паде и эффективное аналитическое продолжение степенного ряда // УМН. 2002. Т. 57. №1. С. 45-142.

7. Геронимус $\mathcal{A}$. Л. О полиномах, ортогональных на круге, о тригонометрической проблеме моментов и об ассоциированшых с нею функциях типа Каратеодори и Шура // Матем. сб. 1944. Т. $15(57)$. С. 99-130.

8. Ахиезер Н.И. О полиномах, ортогональных на дуге окружности // Докл. АН СССР. 1960. T. 130. C. $247-250$.

9. Khrushchev S. Schur's algorithm, orthogonal polynomials and convergence of Wall's continued fractions in $L^{2}(\mathbb{T}) / /$ J. Approx. Theory. 2001. V. 108. P. 161-248.

10. Томчук Ю.Я. О многочленах, ортогональных на заданной системе дуг единичной окружности // Докл. АН СССР. 1963. Т. 151. С. 55-58.

11. Golinskii L. Akhieser's orthogonal polynomials and Bernstein-Szegö method for a circular arc // J. Approx. Theory. 1998. V. 95. P. 229-263.

12. Widom $H$. Extremal polynomials associated with a system of curves in the complex plane // Adv. Math. 1969. V. 3. P. 127-232.

13. Anтекарев А.И. Асимптотические свойства многочленов, ортогональных на системе контуров, и периодические движения цепочек Тода // Матем. сб. 1984. Т. 125 (167). C. 231-258.

14. Peherstorfer F. Elliptic orthogonal and extremal polynomials // Proc. London Math. Soc. (3). 1995. V. 70. P. 605-624.

15. Lukashov A. L., Peherstorfer F. Automorphic orthogonal and extremal polynomials // Canad. J. Math. 2003. V. 55. P. 576-608.

16. Рахманов Е. А. О сходимости диагональных аппроксимаций Паде // Матем. сб. 1977. T. $104(146)$. C. $272-291$.

17. Geronimo J.S., Johnson R. An inverse problem associated with polynomials orthogonal on the unit circle // Comm. Math. Phys. 1998. V. 193. P. 125-150.

18. Peherstorfer F. On Bernstein-Szegö orthogonal polynomials on several intervals // SIAM J. Math. Anal. 1990. V. 21. P. 461-482.

19. Peherstorfer F., Steinbauer $R$. Orthogonal polynomials on arcs of the unit circle. I // J. Approx. Theory. 1996. V. 85. P. 140-184. 
20. Peherstorfer F., Steinbauer R. Orthogonal polynomials on arcs of the unit circle. II. Orthogonal polynomials with periodic reflection coefficients // J. Approx. Theory. 1996. V. 87. P. 60-102.

21. Barrios Rolanía D., Lopez Lagomasino G. Ratio asymptotics for polynomials orthogonal on arcs of the unit circle // Constr. Approx. 1999. V. 15. P. 1-31.

22. Akhieser N. I. Über einige Funktionen, die in gegebenen Intervallen am wenigsten von Null abweichen // Изв. Казанского физ.-матем. об-ва. 1928. Т. 3. № 3. С. 1-69.

23. Лукашов А. Л. Алгебраические дроби Чебышёва-Маркова на нескольких отрезках // Anal. Math. 1998. T. 24. C. 111-130.

24. Belokolos E.D., Bobenko A.I., Enol'skii V.Z., Its A.R., Matveev V.B. Algebro-geometric approach to nonlinear integrable equations. Berlin: Springer-Verlag, 1994.

25. Богатырёв А. Б. Об эффективном вычислении многочленов Чебышёва для нескольких отрезков // Матем. сб. 1999. Т. 190. №11. С. 15-50.

26. Baker H.F. Abelian functions. Abel's theorem and the allied theory of theta functions. Cambridge: Cambridge Univ. Press, 1995.

27. Burnside W. Further note on automorphic functions // Proc. London Math. Soc. (3). 1892. V. 23. P. 281-295.

28. Burnside W. On a class of automorphic functions // Proc. London Math. Soc. (3). 1892. V. 23. P. $49-88$.

29. Геронимус $Я$. Л. Полиномы, ортогональные на круге, и их приложения // Записки Ин-та математики и механики и Харьковского матем. об-ва. 1948. Т. 19. С. 35-120.

30. Peherstorfer F., Steinbauer $R$. Strong asymptotics of orthonormal polynomials with the aid of Green's function // SIAM J. Math. Anal. 2000. V. 32. P. 385-402.

31. Peherstorfer F. Orthogonal and extremal polynomials on several intervals // J. Comput. Appl. Math. 1993. V. 48. P. 187-205.

32. Saff E. B., Totik V. Logarithmic potentials with external fields. Berlin: Springer, 1997.

33. Peherstorfer F. Deformation of minimal polynomials and approximation of several intervals by an inverse polynomial mapping // J. Approx. Theory. 2001. V. 111. P. 180-195.

34. Totik V. Polynomial inverse images and polynomial inequalities // Acta Math. 2001. V. 187. P. 139-160.

35. Чандрасекхаран К. Введение в аналитическую теорию чисел. М.: Мир, 1974.

36. Дубровин Б. А. Римановы поверхности и нелинейные уравнения. Ижевск: НИЦ Регулярная и хаотическая динамика, 2001.

37. Teschl G. Jacobi operators and completely integrable nonlinear lattices. Providence, RI: Amer. Math. Soc., 2000.

38. Форд Л. Р. Автоморфные функции. М.-Л.: ОНТИ, 1936.

39. Peherstorfer F., Steinbauer R. Perturbation of orthogonal polynomials on the unit circle - a survey // Proc. Workshop Orthogonal Polynomials on the Unit Circle / ed. M. Alfaro et al. Leganés: Universidad Carlos III, 1994. P. 97-119.

40. Лукашов А. Л. Многочлены, ортогональные на нескольких дугах // Современные проблемы теории функций и их приложения. Тезисы докладов 10-й Саратовской зимней школы. Саратов: Изд-во Саратовского ун-та, 2000. С. 83-84.

41. Лукашов $А$. Л. Круговые параметры многочленов, ортогональных на нескольких дугах единичной окру жности // Теория приближения функций и операторов. Тезисы докладов Международной конференции, посвященной 80-летию со дня рождения С.Б. Стечкина. Екатеринбург: УрГУ, 2000. С. 95-97.

42. Magnus A. Recurrence coefficients for orthogonal polynomials on connected and non connected sets // Padé approximation and its application. Berlin: Springer, 1979. P. 150-171. (Lecture Notes in Math. V. 765.)

43. Lukashov A.L., Peherstorfer F. Zeros of polynomials orthogonal on two arcs of the unit circle // (submitted to J. Approx. Theory). 